\title{
Large-Scale Architectural Asset Extraction from Panoramic Imagery
}

\author{
Peihao Zhu, Wamiq Reyaz Para, Anna Frühstück, John Femiani, and Peter Wonka
}

\begin{abstract}
We present a system to extract architectural assets from large-scale collections of panoramic imagery. We automatically rectify and crop parts of the panoramic image that contain dominant planes, and then use object detection to extract assets such as façades and windows. We also provide various tools to identify attributes of the assets to determine the asset quality and index the assets for search. In addition, we propose a UI to visualize and query assets. Finally, we present applications for urban modeling and texture synthesis.
\end{abstract}

Index Terms - asset extraction, urban modeling, object detection, clustering, semantic segmentation.

\section{INTRODUCTION}

In this work, we present a system for extracting a library of image patches that depict architectural elements (e.g., façades, windows, doors, or balconies) from a large collection of panoramic images. Ideally, we would like to collect enough data to cover the design space of architectural elements in different regions around the world. This data has the potential to enable applications in architectural analysis, urban modeling, encoding priors for architectural reconstruction, and geolocalization. Existing collections of assets do not have the same geospatial diversity, or are biased towards non-geotypical examples (e.g., Flickr images are biased toward popular landmarks). Fig. 1 shows an overview of our system .

For example, how many different visual classes of window are there? A modeler tasked with creating a procedural model of Paris would need to know what the most frequent window types are in order to create $3 \mathrm{D}$ window models of the window types. In addition, the modeler might be interested in knowing where to find representatives of these different window types close to their office to create laser scans. Furthermore, many modeling algorithms based on machine learning require a large amount of data. For example, training a state-of-the-art Generative Adversarial Network (GAN) [1], [2], [3], [4] on façade images requires more than 10,000 examples, but the largest existing façade datasets such as those presented in [5], [6] have fewer than 1000 façade images.

The asset extraction problem is closely related to object detection. The distinguishing characteristic is that we are dealing with panoramic images and that the image regions that contain architectural elements must be rectified. Thus, we are dealing with sequential object detection and rectification problems. To this end, we begin by extending the very effective horizon-line-first approach adopted by Simon et

- P. Zhu is with KAUST, E-mail: peihao.zhu@kaust.edu.sa.

- W. R. Para is with KAUST, E-mail: wamiq.para@kaust.edu.sa.

- A. Frühstück is with KAUST, E-mail: anna.fruehstueck@kaust.edu.sa.

- J. Femiani is with Miami University, E-mail: femianjc@miamioh.edu.

- P. Wonka is with KAUST, E-mail: pwonka@gmail.com. al. [7] for extracting and rectifying dominant façade planes in the panoramic image. The method presented in Simon et al. [7] works well on images that are the result of a pinhole projection; however, here we propose a novel process that can be applied to an entire spherical panorama. Second, we employ a standard object detection framework [8] to extract assets, such as façades, windows, and doors. Third, we propose a collection of neural networks and algorithms to extract important attributes about the assets. The attributes can be helpful in assessing image quality, e.g., blur, viewing angle, or occlusions. They also support search and analysis tasks, e.g., retrieving images based on the number of floors or percentage of the façade covered by windows. Fourth, we use a simple UI to enable search and visualization tasks. Finally, we demonstrate how to use our system for multiple target applications and provide a quantitative evaluation of our work.

The most closely related system to our work is the extraction of discriminative image patches from panoramic images by the system of "What Makes Paris Look like Paris" (WMPP) by Doersch et al. [9]. We believe that our system has multiple advantages. First, many applications can benefit from extracting image patches that are correctly and semantically cropped. While the system of WMPP extracts patches that cut through elements, e.g., the top right corner of a window and part of the ornament on top, we can directly extract semantically meaningful patches like windows or doors. Second, we enable the extraction of elements at different scales, especially the extraction of complete façades in addition to elements contained inside a façade, such as windows or doors. Third, we provide multiple extensions to enable quality control of the extracted image patches and we gather important information for better search and analysis.

The main contributions of this work are:

- An automatic algorithm for extracting and rectifying dominant façade planes from a panoramic image.

- A completely automatic system for extracting architectural elements from a large-scale panoramic image collection.

- A dataset of 199,723 extracted façade images including the contained windows, doors, and balconies together 


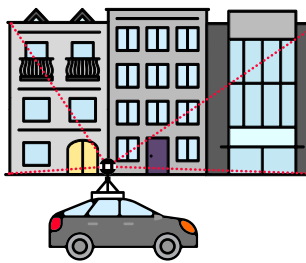

(a) ACQUISITION

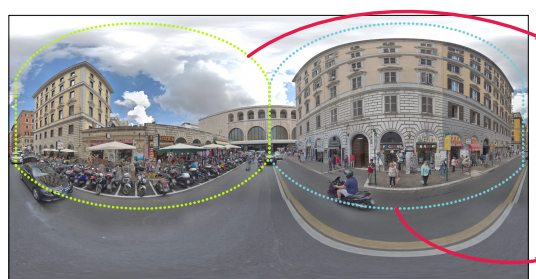

(b) PANORAMA

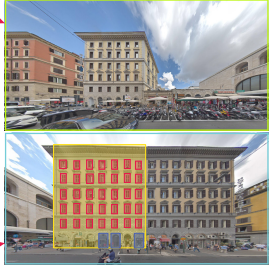

(c) RECTIFIED FAÇADES

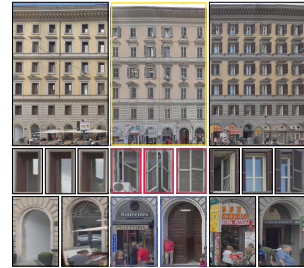

(d) ASSETS

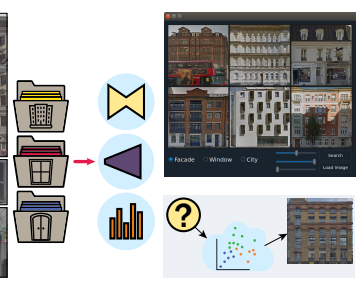

(e) UI \& APPLICATIONS

Fig. 1: System overview. a) We download the panoramic street-level imagery that is acquired at a very large scale by multiple companies. b) We analyze each image to extract and rectify patches that contain façades. c) On the rectified patches, we use object detection to find rectangular image regions that contain architectural elements such as façades, windows, doors, and balconies. d) For each extracted asset (we show a selected subset), we compute multiple attributes by neural networks or traditional algorithms. e) A UI enables visualization and search to use the library of extracted assets for various applications.

with descriptive attributes ${ }^{1}$.

- The demonstration of multiple novel applications enabled by this dataset.

\section{Related Work}

We discuss applications of the proposed pipeline and work that aims to determine meaningful views from panoramas. We can broadly categorize work into systems for visual data mining, approaches for analysis of urban areas based on street-level imagery, urban modeling approaches to create large-scale 3D models of the built environment, and finally, approaches that deal with viewpoint and 6DOF pose estimation of objects from photographs.

\subsection{Visual Photo Collections and Visual Data Mining}

Visual data mining aims to find patterns or to search through large photo collections. AverageExplorer [10] is an example of an approach that enables interactive exploration of image collections through weighted-averaged images, and NEIL (Never Ending Image Learner) [11] trawls the web to discover patterns between images. These approaches apply to arbitrary image collections. Street-level imagery enables other image retrieval applications such as automatic geolocation to estimate the geographic location of a photo on a map with the benefit of training on geotagged images from Flickr, or from Google Street View imagery. Examples of such approaches are found in [12], [13].

\subsection{Visual Urban Machine Learning}

Analysis of architecture imagery can also be used to infer non-visual attributes of urban environments. City forensics [14] used the appearance of buildings (including graffiti, or whether shutters were open or closed) to predict crime rates or affluence. Other attributes of an environment were evaluated as part of the 'Place Pulse' project [15] to predict whether a neighborhood was safe, lively, boring, wealthy, depressing, or beautiful, based on street-level imagery. The proposed work is most similar to [9], who mine Google StreetView data to extract square patches that best discriminate the cities of each panorama. Their approach

1. https://github.com/ZPdesu/lsaa-dataset automatically learned to extract patches that contain windows, signs, or balconies (depending on the city) and they were able to produce style-summaries of different locations. They demonstrated that the appearance of key architectural elements is important for understanding the diversity of urban environments.

\subsection{Urban Modeling}

Asset extraction is an important task for urban modeling. A range of applications such as architecture, city planning, real-estate, path planning for drones, flight simulation, computer games, and movies, benefit from 3D urban models.

Procedural modeling approaches [16], [17] generate 3D building models, often using shape grammars, to construct a solid model and then planting certain assets such as detailed textures, or pre-modeled windows, doors, or balconies from a library. For example, CityEngine [18] relies on a library of reusable and tileable textures (assets) to inject variety and realism into building models.

Urban reconstruction applications rely on inverse procedural modeling and learn structure or parameters of a grammar from images. For example, [19] learn parameters of an L-system from examples and [20] learn a split grammar from 3D models of architecture. The proposed asset extraction system can also provide input for single-image reconstruction approaches [21], [22] in which the parameters of a procedural model are estimated from a single RGB or RGB-D image.

Large-scale asset extraction can help to build prior knowledge about façade structures for machine-learning approaches. One application is façade synthesis using neural networks. GANs such as StyleGAN [4], progressive GAN [23], and BigGAN [3] are capable of generating extremely realistic images but rely on a very large amount of training data. Conditional GANs are able to generate façade images based on layouts, as demonstrated by pix2pix [24]. Other approaches such as bicycleGAN [1], MUNIT [2], and SEAN [25] are able to generate very highly detailed models with label images.

\subsection{Pose Extraction and Rectification}

The 6DOF pose estimation problem has a long history, and deep-learning approaches have been developed to solve the 


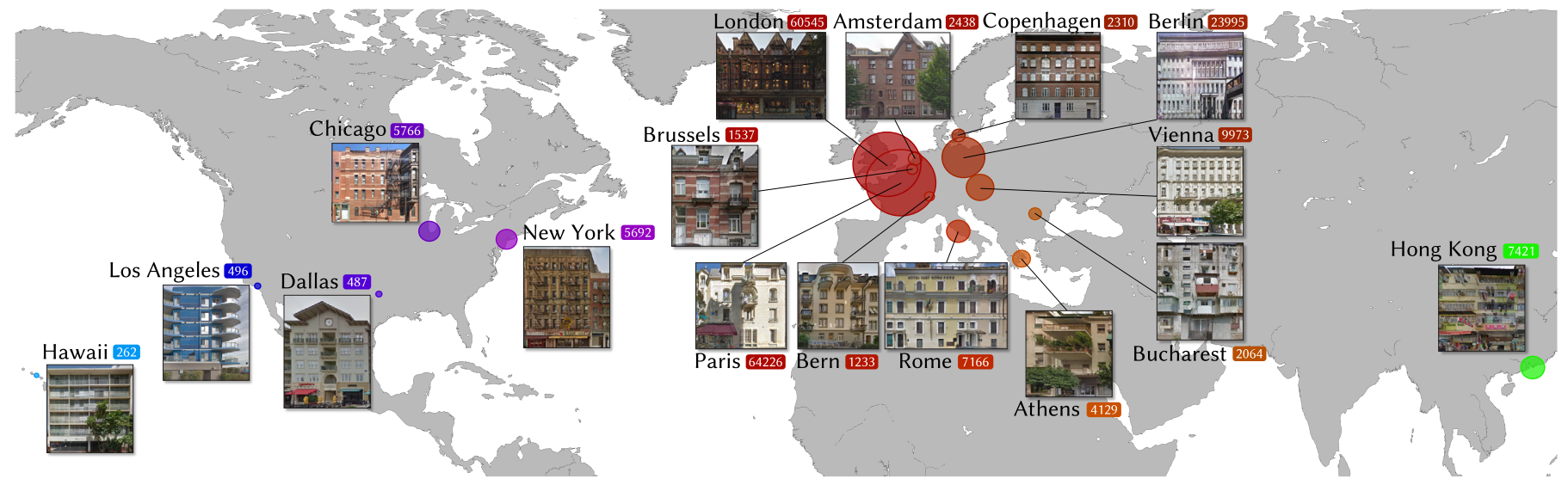

Fig. 2: We show the geographic origins of the 78,377 photospheres that we aggregated from locations across three continents.

problem by training on synthetic data [26], predicting the eight 2D key-points that form the corners of a 3D cube [27], or by directly predicting location and orientation [28].

The idea that many man-made structures tend to be rectangular, such as architectural elements, has been exploited for some time. Early approaches would group edges to find rectangles on façades [29], [30], [31]. Most recent approaches target Manhattan scenes. A Manhattan scene has only three Vanishing Points (VPs) corresponding to orthogonal directions. Under such an assumption, it is sufficient to identify VPs corresponding to a zenith direction and a horizontal direction. Images can then be reprojected by a homography that maps those directions to the image's cardinal axes in order to rectify it. Once rectified, rectangular bounding boxes of objects can be found using an object detector. Instead of just using edges or other low-level primitives, Fond et al. [32] describe higher level features that incorporate prior information about urban scenes, such as symmetry or rectangular shape. The pipeline in Fond et al. is two-step; the initial proposals come from a segmentation approach based on contours. In the second step, the more expensive higherlevel features are determined. This is a common theme in object detection; see, for example, [33], [34]. Our pipeline also follows a two-stage process. The rationale for using a two-stage process is described in Section 3.3.4. However, their method operates on pinhole projected images and assumes a Manhattan world, whereas our approach allows a much wider variety of building orientations and operates on panoramic imagery.

Affara et al. [35] rectifies façade images using RANSAC to estimate a homography that maps a large number of edges to vertical or horizontal lines. Wu [36] uses symmetry of repeated structures on a façade (in addition to VPs) to rectify façade images. The Video Compass [37] uses Expectation Maxmization (EM) to cluster the intersections of pairs of lines. Other approaches use different clustering methods to group VPs; for example J-Linkage [38] or RANSAC [39]. Tretyak et al. [40], [41] adopt an Atlanta world [7] assumption instead of the more common Manhattan world assumption. In an Atlanta world, planes share a common Zenith Point (ZP) and a horizon line that may contain many VPs.

Several attempts have been made to use deep learning for detecting VPs in images. Zhai et al. [42] used AlexNet to predict the horizon line as an angle and distance from the principal point. A more recent approach uses deep learning to assign scores to a Gauss sphere [43]. Similar to [44], the authors used a coarse grid, and so they ultimately rely on EM to refine their estimate.

The current state of the art in rectification of pinholeprojected images is the approach of Simon et al. [7], who observe that the $\mathrm{ZP}$ and the principal point of a pinhole image form a line that is perpendicular to the horizon. They use a projection histogram to identify a meaningful cluster of edges at the horizon line. However, this approach intrinsically relies on the geometric properties of a pinhole projection and cannot directly be applied to panoramic imagery. Panoramic images are addressed by Bazin et al. [45], who assume a Manhattan world. They carry out a grid search through rotation roll, pitch, and yaw until they find a set of Cartesian axes that align with up to three VPs. While the method can be fast if the initial poses are known, the search space can be quite large and the method is limited to a Manhattan world, which is unlikely in panorama images. We build on the approach of Simon et al. [7] in our system by introducing a novel extension that works with panoramic imagery instead of 2D images. The details are given in Section 3.3.

\section{FRAMEWORK}

\subsection{Overview}

Our framework combines multiple components to tackle the asset extraction problem:

1) Data Collection: we extract Panoramic Streetview (PSV) images from the internet (See Section 3.2).

2) Candidate Plane Extraction and Rectification: for each PSV image, we detect candidate planes and rectify them by computing the dominant orientation of the linear features on the plane (See Section 3.3).

3) Object Detection: for each candidate plane, we extract assets using object detection (See Section 3.4).

4) Attribute Computation: for each extracted asset, we compute a list of attributes (e.g., resolution, image quality, estimated size in meters, etc.) that are valuable for subsequent processing (see Section 3.5). 
5) User Interface: we provide a UI to visualize, search, and select assets (See Section 3.6).

6) Applications: extracted assets can be used for architectural analysis, procedural modeling, and texture generation using GANs.

In the following subsections, we will describe the individual components in more detail. Our illustrations and exposition will use façades and windows as the most important assets to be extracted, but other façade elements can be extracted with the same framework. See the supplementaty material for additional examples.

\subsection{Data Collection}

A key aspect of the proposed work, similar to [9], is to leverage a large number of geolocated panoramic street level images (photospheres) such as those available using Google Street View [46]. Photospheres are often the result of stitching together a set of overlapping pinhole projected images - a process that can result in some loss of accuracy. Beyond that, typical Geographic Positioning System (GPS) and compass systems have limited precision, especially when mounted on a moving vehicle. As a result, each photosphere possibly has some error in heading, tilt, and roll. We collect data within different geographic regions by first locating buildings using the OpenStreetMap (OSM) API. For each building, we select panoramas within 40 meters. To date, our data is extracted from 78,377 photospheres from 17 cities in 13 countries (See Fig. 2).

\subsection{Candidate Plane Extraction and Rectification}

Panoramic street view images contain many façades, each with a dominant plane, and within each façade there are other assets that tend to share the same orientation. Our system relies on the imagery to select meaningful viewing directions and then crops the viewport around assets in the imagery. We extend the "Horizon Line First" approach for rectification [7] to work on a new class of images, i.e., panoramic photospheres. We first list each step of the process and then explain them in more detail:

1) Estimate the horizontal plane and zenith directions in the panoramic image (Section 3.3.1).

2) Select meaningful VPs on the horizon and then choose candidate viewing directions based on these VPs (Section 3.3.2).

3) For each candidate viewing direction, project an image and confirm the VP estimation based on its contents (Section 3.3.3).

4) Crop projected images to individual façades in each viewing direction (Section 3.3.4).

5) Refine the location of the zenith and VPs based on a single façade (Section 3.3.5).

\subsubsection{Zenith Estimation}

We assume an Atlanta world model for urban scenes in which there is a single dominant up (or zenith) direction shared by all façade planes. Approaches such as that applied in [7] are very robust at estimating the zenith direction from pinhole-projected images by clustering the intersections of near-vertical lines. Line detection is an important step in this process; however, the spherical projection used by panoramic images distorts lines. Our proposed solution is to detect candidate zenith directions using $K_{\text {vdirs }}$ pinhole images that cover the panorama (See Fig. 4). Each potential zenith direction corresponds to a point on the photosphere, yielding a number of candidate ZP (See Fig. 6). Note that we will use ZP and VP to indicate either points on a sphere, $3 \mathrm{D}$ unit vectors, or points in images interchangeably.

To illustrate the process, an input panoramic image is shown in Fig. 3 and corresponding projected images are shown in Fig. 4. Fig. 5 shows the candidate horizon lines.

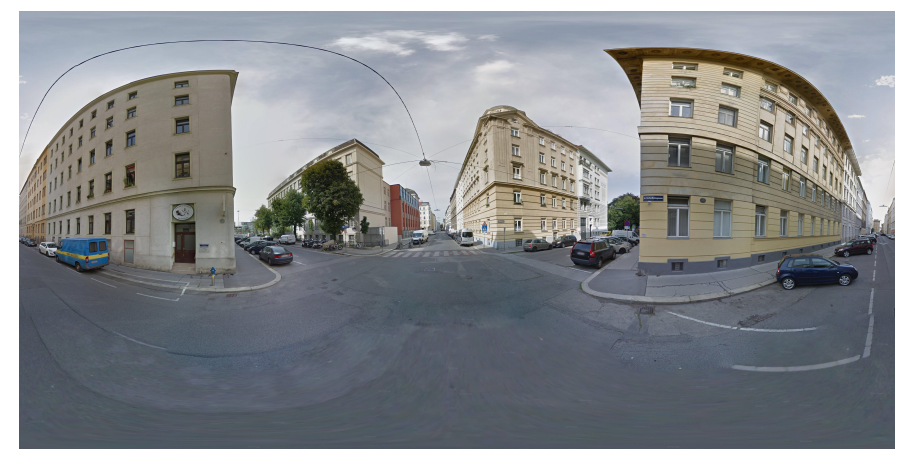

Fig. 3: The panoramic images used in our work, shown with a spherical/equirectangular projection, have a resolution of $13312 \times 6656$ pixels.

The next step is to find a consensus $\mathrm{ZP}$ of the panoramic image based on multiple candidate ZPs. We consider two approaches for determining the consensus $\mathrm{ZP}$ : the robust mean and the $S V D$ with outlier removal. The robust mean is found by first taking the mean of the ZP estimates, and then projecting back onto the sphere. The nearest $K_{\text {inlier }}$ points to the mean are then retained and the process is repeated to form a final estimate. The SVD approach works similarly, except the consensus vector $\hat{\mathbf{x}}$ is chosen as

$$
\hat{\mathbf{x}}=\arg \max _{\|\mathbf{v}\|=1} \sum_{k=1}^{K_{\text {inlier }}}\left|\mathbf{v}^{T} \mathbf{x}_{k}\right|^{2},
$$

where $\hat{\mathbf{x}}$ is the consensus point and $\mathbf{x}_{k}$ is the $k^{\text {th }} \mathrm{ZP}$. The solution $\hat{\mathbf{x}}$ is the largest eigenvector of $\sum_{k} \mathbf{x}_{i} \mathbf{x}_{i}^{T}$.

\subsubsection{Vanishing Point Estimation}

We use an Atlanta world assumption [7] that all VPs except the zenith are at the horizon. To find the VPs, we return to each of the pinhole-projected images and select every line segment that is within $15^{\circ}$ (the same threshold used by [7]) of the horizon. The homogeneous coordinates of the intersection between each line segment and the horizon form points on a sphere (see Fig. 5, middle row). We rotate the photosphere so that the zenith is up, and record the heading of each rotated horizon point (in degrees). Following [7], candidate VPs are detected by finding the Max Meaningful Modes (MMMs) [47] of the histogram of the headings. Antipodal VPs on the photosphere correspond to the same plane; thus the histogram is periodic with a periodicity of $180^{\circ}$. Note that in the method of Simon et al., care must be taken when searching for a histogram to account for the fact that a uniform distribution of directions would 

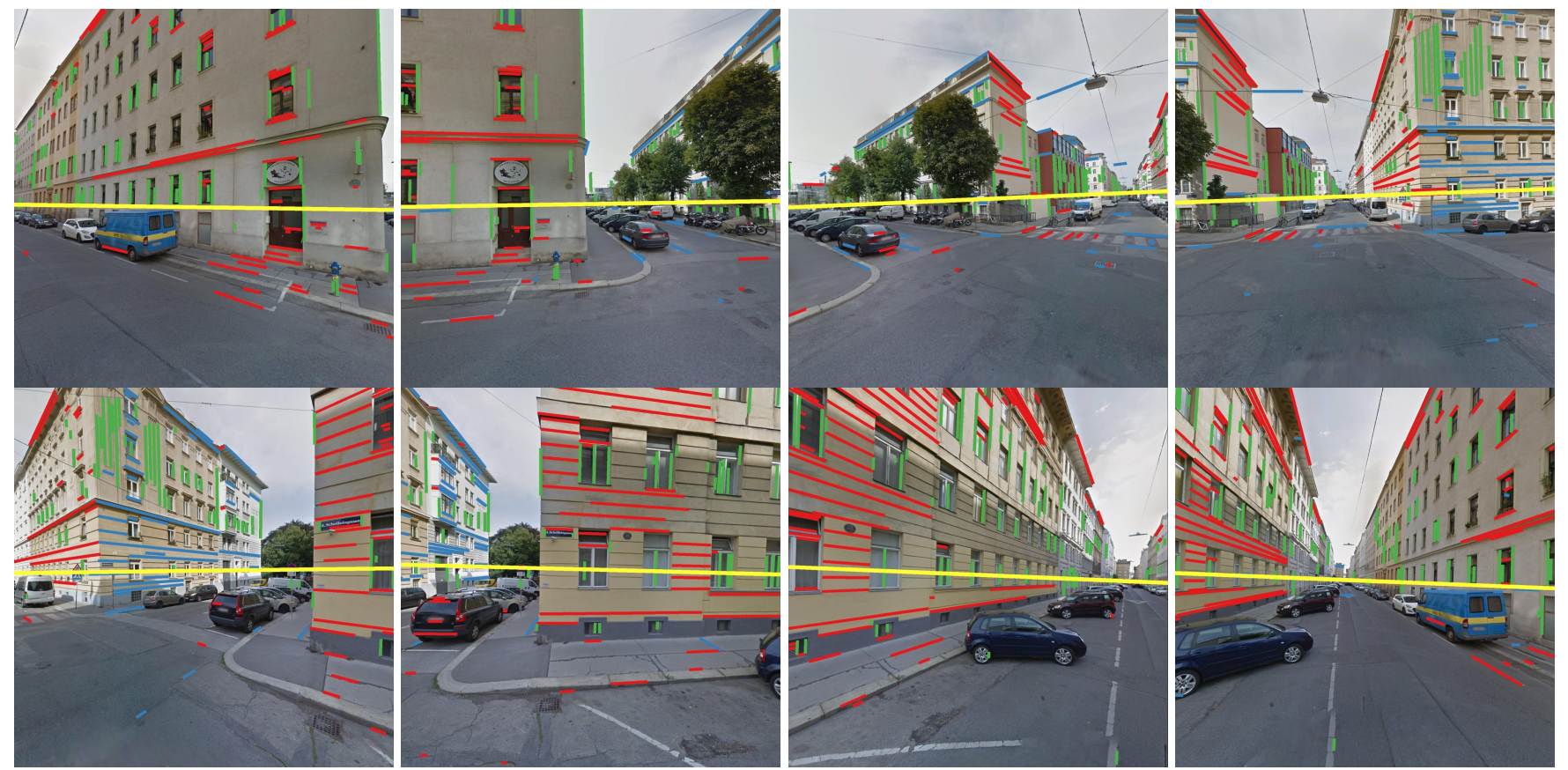

Fig. 4: We show 8 projected candidate viewing directions. Each one has a 90 degrees vertical and horizontal FOV and a 45 degree horizontal overlap with the neighbouring image. Green line segments: line segment groups that converge to the ZP. Red and Blue line segments: line segment groups for different VPs in one image. Yellow line: estimated horizon line for each candidate viewing direction.

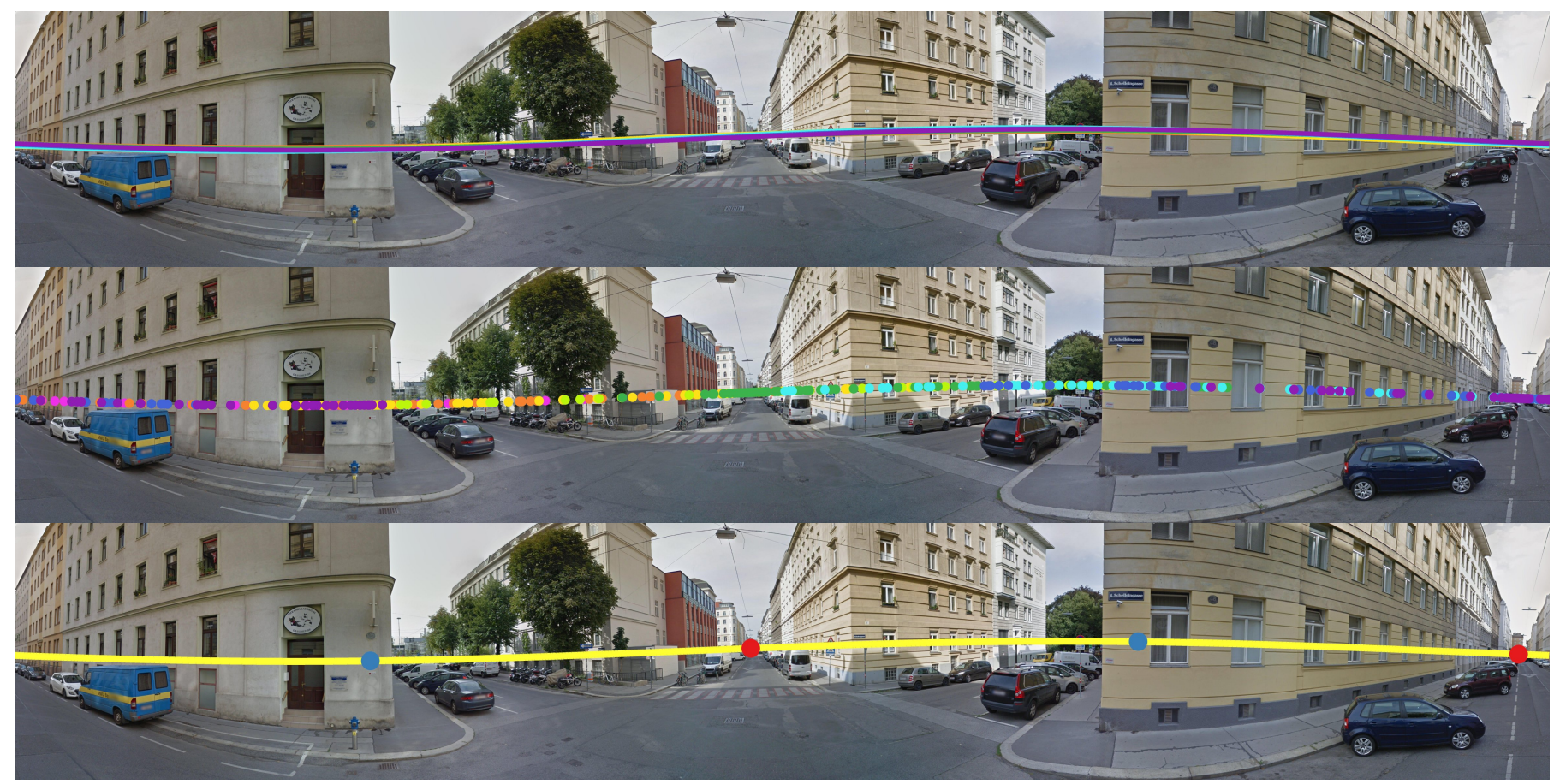

Fig. 5: Top: Horizon line estimates for each of the viewing directions are shown in different colors - the horizon is the circle of points that are orthogonal to the consensus zenith direction. Middle: Each point is the intersection of a horizontal line and a great circle where the photosphere intersects the horizon plane. Different colors correspond to potential VPs from different candidate planes. Bottom: Red dots: antipodal points from the first VP, Blue dots: the second VP, Yellow curve: horizon line. The same color means the same VP with a periodicity of $180^{\circ}$. In this example, the blue dots correspond to a single VP for planes parallel to the road, whereas the red dots result from the visible sides of those buildings (one near the center of the panorama and another at the end of the street). 
form a nonuniform distribution of points on the horizon line. Because our histogram is circular, we are able to space the bins equally, which simplifies finding of the mode. The MMMs identified using this histogram are shown in Fig. 7 and Fig. 5 (bottom). To calculate more precise VPs, we use the largest singular vector of all of the points within the selected bin.

\subsubsection{Vanishing Point Refinement}

The next step is VP refinement. Each estimated VP corresponds to two potential viewing directions (e.g., align the VP with the camera's positive or negative $x$-axis). It is possible that a façade exists in only one of these directions, or that the planes in either direction are not perfectly parallel. We render this pair of pinhole-projected images with a wide horizontal field of view, $K_{\mathrm{hFOV}}$. In each image, we estimate new VPs using only the contents of the projected image. If the VP estimated from the projected image and the VP predicted from the histogram are further than a distance threshold, $K_{\mathrm{vdist}}$, then the viewing direction is discarded. We use a threshold of $K_{\text {vdist }}=5^{\circ}$, which exceeds the largest difference between the true and predicted viewing directions that we observed in the validation data. Each image would ideally have the same VPs, but a combination of non-parallel building facades and panorama stitching artifacts mean that this is not always the case, and, as shown in Table 2, performing refinement improves the rectification quality.

\subsubsection{Façade Cropping}

After the VP/ZP refinement, we have a set of pinholeprojected images each with wide FOV (e.g., $K_{\mathrm{hFOV}}=$ $\left.[-75,75], K_{\mathrm{vFOV}}=[-40,80]\right)$. Each image is chosen based on a dominant plane, but there may be several distinct building façades within the plane. We use the FasterRCNN [8] object detector and train it on façade annotations from [48], [49] to determine the bounding boxes of each façade. The training set for the Faster-RCNN model is obtained as follows: we produce approximately rectified images using building GIS data and the panorama's GPS metadata. Annotators further refine the image by annotating the four corners of the main façade, following which a homography is estimated, which transforms the quadrilateral formed by the four corners into a rectangle. Annotators then label the window elements on this rectified façade. We train the system using bounding boxes of the annotated façades as well as the window elements. We add windows as an object detection target because our experiments show that window detection improves accuracy in façade detection (See Table 5).

\subsubsection{Façade Refinement}

Façades on the same side of the street are often nearly, but not exactly, parallel to each other. In order to select a viewing direction for each façade more precisely, we re-estimate the vanishing points in sub-images that are cropped to the bounding box of each façade using Simon's method [7]. The facade image is then warped and cropped again to obtain a final rectified image.

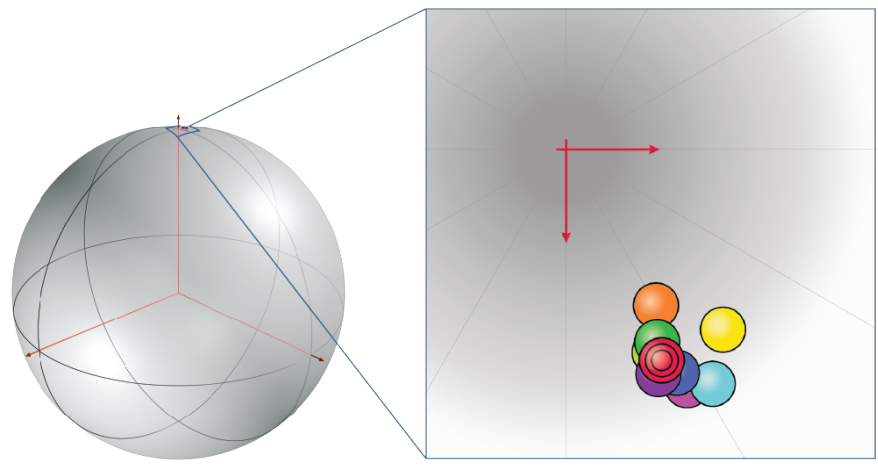

Fig. 6: We show eight detected ZP candidates, each obtained from a single viewing direction (projected image). While all candidate points are close to the ZP estimated in the original data (left), our refinement achieves important improvements by removing outliers, as shown in the inset (right). The red point marked by three circles is the consensus $\mathrm{ZP}$ computed by SVD.

The result of this process is that each individual detected façade in each photosphere has an associated viewing direction and viewport that can be used to extract a rectified façade image. These rectified images are assets themselves, and they are also used to detect sub-façade elements such as windows and doors.

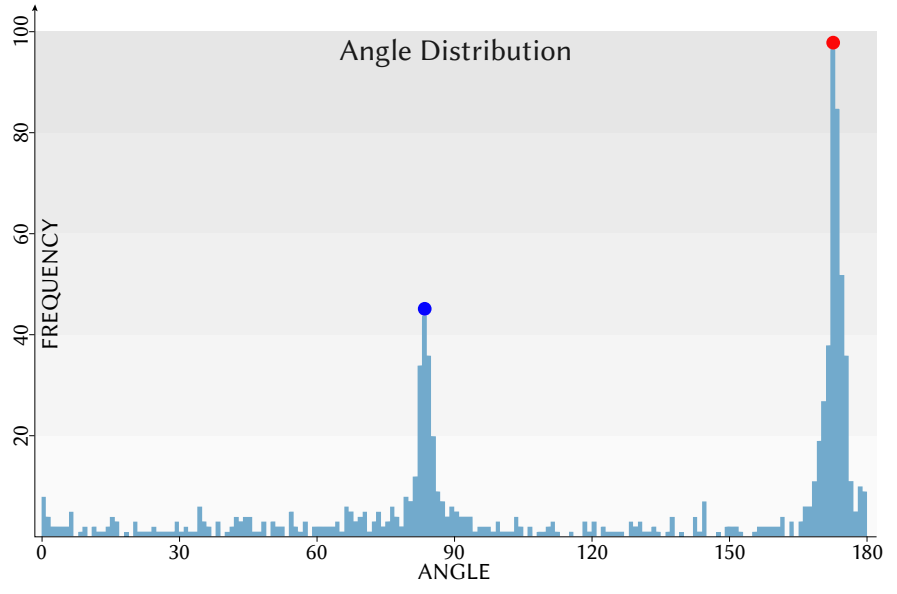

Fig. 7: The red and blue dots are the candidate VPs corresponding to the panorama of Fig. 5. Here the red VP is around $172.5^{\circ}\left(352.5^{\circ}\right)$ degrees and the blue VP is around $83.5^{\circ}\left(263.5^{\circ}\right)$.

\subsection{Detection Framework}

Once a viewing direction and viewport for each façade has been determined, we wish to identify parts that comprise the façades, such as windows, doors, and balconies. To achieve this, we use the same training set described in Section 3.3.4, but split it into cropped images for each façade. In the original training set, some façades were marked as 'unlabeled' to indicate that sub-elements were not annotated. For the remaining façades, we extracted a sub-image from the 
original data to form a new set of 1434 annotated singlefaçade images. We used a random split of $80 \%$ for training and used $20 \%$ of the data to evaluate asset detection. We used the bounding boxes of each asset as training data for Faster-RCNN [8] and trained it to detect each type of asset listed above.

Faster-RCNN [8] was configured with its default settings. ResNet101 [50] was used as a backbone network and a FPN module [51] was used for multi-scale detection. Training using SGD for 90,000 iterations took 48 hours on 4 RTX 2080 Ti GPUs. During training, each image was rescaled so that its height was 800 pixels and the width of each image was clipped at 1333 pixels (the default setting).

\subsection{Attribute Computation}

We leverage the large amount of data gathered from our pipeline to enable analysis, filtering, and searching of the extracted assets. The attributes described in this section are used in a graphical UI that is described in Sec 3.6. We broadly divide attributes into five categories. Metadata attributes are derived from OSM metadata and the images themselves. Homography attributes are derived from the rectification process. Semantic attributes are based on trained models. Quality attributes are indicators of the image quality of the asset. The final category is a Semantic Embedding found by the unsupervised clustering method of [52].

\subsubsection{Metadata Attributes}

The assets we capture are extracted from panoramas that carry metadata including geographic properties, such as the country, city, building, latitude, longitude, and compass orientation information. We also include information on the sub-image that contains the asset, such as the aspect ratio, which is the width divided by the height of the asset's bounding box. The resolution of an asset depends somewhat on the rectification process, but we record the width and the height of the projected view of each asset.

\subsubsection{Homography Attributes}

The result of our rectification process described in Section 3.3 is a viewport from a pinhole-image that is often far from the camera's optical center. As a result, many assets exhibit exaggerated perspective effects, and protruding or recessed features of the façade result in occlusion. The view angle is the angle between the viewing direction and a vector pointing at the center of the asset. The smaller the absolute angle, the smaller the risk of self-occlusion or other image distortions.

\subsubsection{Semantic Attributes}

Semantic attributes are estimated properties of the asset and are predicted by either training deep networks for each attribute, or recording the geographical metadata associated with the panorama the asset came from. Since each asset is a part of a façade, we record the number of windows in the façade that contains the asset.

In order to predict the number of floors of each façade, we manually labeled 5000 images and trained a ResNet101 [50] classification network to estimate the number of floors of each building. We built a classifier with nine classes, eight of which indicate the actual number of floors, with a special label for images that do not have a meaningful façade. Another special label is given to all façades with $0,1-7$, and 8 or more floors.

We trained the DeepLab V3+ [53] semantic segmentation network on façade elements such as windows, balconies, and also ground-level obstructions such as vehicles or trees. This network is then used to estimate the area occupied by the various façade elements such as window area, balcony area, door area, etc.

\subsubsection{Quality Attributes}

A common degradation in our collected assets is the amount of blur that can result from elements that are undersampled in the panorama. Even blurred images may be suitable for some applications, for example in mass models [49], [54], while more detailed close-ups would require very sharp assets. To allow a designer to select assets with varying sharpness, we estimate the blur using the variance of the Laplacian [55]. The lower the value, the more blurred the image. Another kind of degradation is the presence of occlusion by elements that lie in front of the assets, such as trees, poles, or ground-level obstructions including pedestrians or cars. For each asset, we use the semantic segmentation results from Section 3.5.3 to estimate the fraction of the pixels within its bounding box that may occlude it.

\subsubsection{Embedding}

We use the method of [52] to generate a 4096-dimensional embedding for each of our assets. [52] is an unsupervised training method to extract deep features. This method requires zero supervision and is suitable for our pipeline because, apart from the meta-data, we do not have semantic labels for our assets. The method performs a forward-pass through a network such as the VGG-16[56] to generate a fixed-length representation. Backpropagation is performed by clustering this representation, and then using the assigned cluster labels as pseudolabels and computing the cross-entropy loss. We use the default hyperparameters for learning rate and weight decay, using a batch size of 64 . The number of clusters $(k)$ set during training is also a hyperparameter and the original implementation, trained on ImageNet, uses $k=10,000$, which is an order of magnitude larger than the number of classes in ImageNet. In their analysis, [52] found that setting $k$ to be larger than the number of expected classes led to improved learned embedding. We use $k=200$ because we believe that this number of clusters is large enough to capture the variation in façade designs. Note that once the training process is complete and we get the embedding vectors for our assets, we are free to use a different clustering algorithm and a different $k$.

We use this embedding for two major purposes: a) visualizing the distribution of façades and b) performing a similarity search in the space of assets. Since it is difficult to perform either of these tasks in a relatively highdimensional space, we use PCA to project this embedding onto 200 dimensions. For visualization, we use UMAP[57] to further reduce the embedding to 2 dimensions. We use the $L 2$ distance on the 200 dimensional embedding to perform the search. 


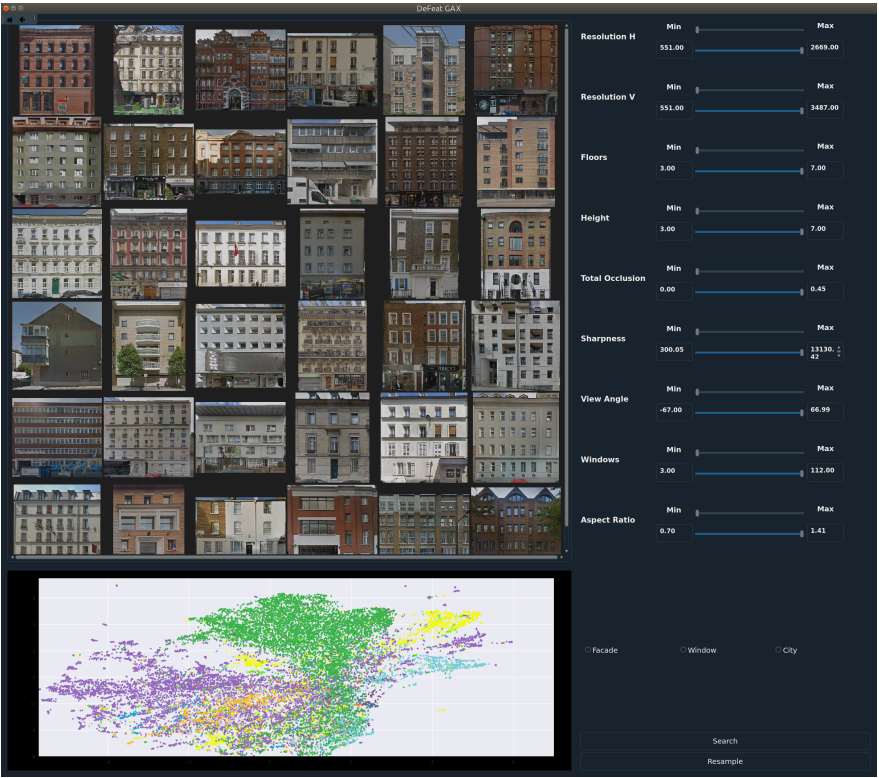

Fig. 8: The interface to interactively filter assets and traverse semantically similar asset-clusters. The filters on the right are the asset attributes. A two-dimensional representation of the assets fills the left pane.

\subsection{User Interface}

A key component of our system is a UI for interactive asset exploration and selection. A number of very effective approaches for image exploration exist; we cite AverageExplorer [10] as a tool that allows interactive exploration and query over sets of large images, and Photo Tourism [58] as UI for exploring geotagged photographs in 3D with capabilities that include locating new points of view, or higher resolution imagery of the same object.

We consider a UI for asset extraction to have three critical functions. The first is to explore the space of asset images, the second is to filter the collected assets according to their attributes, and the third function of the UI is to search for assets that are similar to a query. The key operation of our UI is to filter out samples based on attributes or their distance from query images, and then to use $k$-means on the 200dimensional embedding of each asset to identify $K_{\text {summary }}$ distinct cluster representatives, that are shown in a grid (Fig. 8). Users can interactively drill-down on a cluster by clicking on it, which filters out assets from all other clusters and then repeats the clustering step on the surviving assets. Filtering based on attributes is accomplished via widgets that allow users to filter assets by selecting ranges of values per attribute. For example, by adjusting the maximum viewing angle, it is possible to constrain results to assets that are captured from less oblique viewpoints. A second example of filtering is adjusting the maximum amount of occlusion to retrieve only the assets that are unobstructed by trees or vehicles. The third function of the UI is to search for an example based on a query image. To accomplish this, a user provides a query image of a single asset and assets from the dataset are returned in ascending order of their distance from the query in the 200-dimensional embedding space.

\section{Results and Applications}

In this section, we present quantitative and qualitative evaluations of our system.

\subsection{Quantitative Evaluation}

The first quantitative evaluation is an analysis of the rectification and viewport estimation method described in Section 3.3. A key challenge we face was that we could not manually identify a single ZP for an entire panorama, so instead we selected a sample of semi-automatically rectified façades from the dataset of [48], choosing 52 panoramic images. The data was already partially rectified based on Geographic Information System (GIS) information on the location and orientation of building walls. Within these images, annotators marked the most clearly visible vertical and horizontal lines of each façade. These markings were then used to find the target homography that was used to rectify each façade image. The images we used had coarse polygonal annotations for other features, which we warped using the same homography to derive bounding boxes. The dataset contains marked ground truth regions for 409 façades, 4501 windows, 451 doors, and 370 balconies. We also have labels for 8141 other architectural elements, but we do not focus on those during our evaluation.

Existing rectification approaches use a variety of metrics: [7] uses the maximum deviation (within the image) from a horizon line. However, this is difficult to apply to cropped regions. Viewpoint estimation methods [28], [27] use the ADD metric that measures the average distance between $3 \mathrm{D}$ keypoints on the original and predicted transformations, as well as $5 \mathrm{~cm}, 5 \mathrm{deg}$ [59], which measures the fraction of results that are within $5 \mathrm{~cm}$ and $5^{\circ}$ of the target objects.

We do not have true 3D ground-truth annotations, nor do we have reliable horizon lines within the images in general, so we propose the following metric based on the directions of vertical and horizontal lines that are marked in the ground-truth.

In our annotations, each image has four lines, and we let $\alpha_{i}$ indicate the angle between the $i^{\text {th }}$ ground-truth line (after re-projection using the predicted homography) and the corresponding vertical or horizontal direction; see Fig. 9:

$$
\begin{gathered}
\bar{\alpha}=\left(\left|\alpha_{1}\right|+\left|\alpha_{2}\right|+\left|\alpha_{3}\right|+\left|\alpha_{4}\right|\right) / 4 \\
\max \alpha=\max \left(\left|\alpha_{1}\right|,\left|\alpha_{2}\right|,\left|\alpha_{3}\right|,\left|\alpha_{4}\right|\right)
\end{gathered}
$$

We aggregate this metric over the entire dataset to define four quantities:

- maa: The mean value of $\bar{\alpha}$ over the test set.

- mma: The mean value of $\max \alpha$ over the test set.

- poa: The percentage of $\bar{\alpha} \leq 1^{\circ}$ in the test set.

- pom: The percentage of $\max \alpha \leq 2^{\circ}$ in the test set.

Our goal is to minimize maa and $\mathbf{m m a}$, and to maximize poa and pom.

\subsubsection{Comparison of Rectification Methods}

Table 1 shows a comparison of our rectification result with the other methods that operate on panoramas. As baseline methods, we consider two approaches. One relies on the 
TABLE 1: Comparison of Panorama Rectification Methods

\begin{tabular}{ccccc}
\hline Method & maa $\downarrow$ & mma $\downarrow$ & poa $\leq 1^{\circ} \uparrow$ & pom $\leq 2^{\circ} \uparrow$ \\
\hline GIS & $1.20^{\circ}$ & $2.32^{\circ}$ & $55.77 \%$ & $61.54 \%$ \\
GPS/IMU & $1.15^{\circ}$ & $2.25^{\circ}$ & $53.85 \%$ & $48.08 \%$ \\
Bazin et al. [45] & $0.81^{\circ}$ & $1.66^{\circ}$ & $80.77 \%$ & $80.77 \%$ \\
Ours & $\mathbf{0 . 4 7}^{\circ}$ & $\mathbf{0 . 9 8}^{\circ}$ & $\mathbf{9 4 . 2 3} \%$ & $\mathbf{9 0 . 3 8} \%$ \\
\hline
\end{tabular}

GIS information for a building in order to choose a viewing direction. The other (GPS/IMU) relies on the heading information metadata associated with each panorama in order to select four viewpoints (left, front, right, and back), as was done in WMPP [9] with only two viewing directions. A current state-of-the-art method is proposed by Bazin et al. [45], which we reimplemented; we provide details on our implementation in the supplementary materials. Our proposed approach outperforms all other methods mentioned in the table.

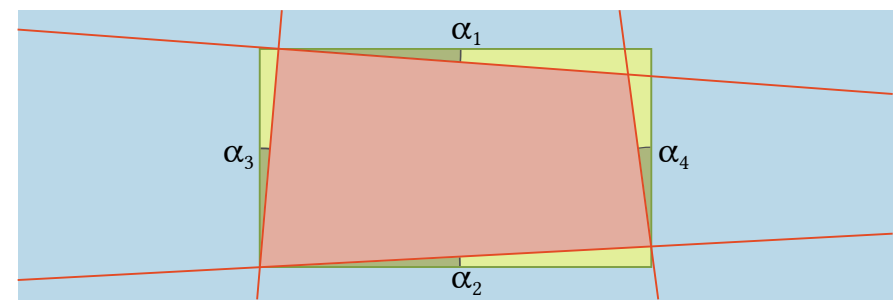

Fig. 9: Slope angle-based homography evaluation. Red line segments are the façade edges of the ground truth façade and the green box shows the horizontal and vertical directions in the rectified image.

\subsubsection{Ablation Study of Rectification}

Table 2 outlines an ablation study of the rectification process described in Section 3.3. In this study, we evaluate different choices for the number of initial pinhole images, $K_{\mathrm{vdirs}}$, as well as the method for choosing the consensus ZP with a different number of inliers, $K_{\text {inliers }}$. The table demonstrates that VP/ZP refinement improves the results, and that façade-based refinement improves them further. When we evaluated façade refinement in Table 2, we treated it as an identity transformation whenever façade detection failed to locate a façade that overlapped the ground-truth region. Façade detection itself is evaluated in Table 3, which shows that we achieve improvements compared to the baseline algorithms. Based on the results, we recommend to use $K_{\text {vdirs }}=16$ view directions for the VP estimation and SVD for computing the consensus ZP with $K_{\text {inlier }}=12$ inliers. We show examples of rectification in Fig. 10.

\subsubsection{Evaluation of Asset Detection}

We evaluated two different detection frameworks: Faster RCNN [8] and Mask RCNN [34] for detecting façades and windows. The results for façades are shown in Table 3 and Table 4 presents evaluation results for detecting windows. We follow established test protocols [33], [8], [34] for object detection and report standard metrics of Average Precision across classes (AP) and Average Recall (AR). These metrics are measured for different settings of the Intersection over
Union (IoU) parameter. For example, an IoU of 50 means that all detections with an IoU greater than 50 are accepted as correct. Furthermore, the IoU setting 50:95 computes the average value by using ten IoU values in the interval 50 to 95 , in steps of 5 . Computing AR requires setting the maximum number of detections, denoted by $\Delta_{n}$ in our results tables.

When evaluating façade detection in Table 3, we found that if we add the windows class as an additional target during training, it helps prevent overfitting and improves the façades detection result, even if the detected windows are not needed by our system. We report the best found settings as F-RCNN*.

For window detection in Table 4, we compare two variations: either detecting the windows directly on rectified images (W on I), or detecting windows on cropped façade images ( $\mathrm{W}$ on $\mathrm{F}$ ). We conclude that the best façade detection is achieved using Faster RCNN and that the results for window detection are similar for Faster RCNN and Mask RCNN.

\subsubsection{Effect of Rectification on Asset Detection}

In Tables 5 and 6, we show the impact of different pipeline settings on the final object detection results for façades and windows. We also evaluate one particular setting of FasterRCNN, i.e., the sizes and aspect ratios of the anchor boxes that are used to generate object proposals. We find that certain classes of architectural elements (e.g., entire façades) exist at fundamentally different scales and aspect ratios to others (e.g., windows or columns). We used the cumulative histograms of scales and aspect ratios of objects in our training dataset to choose three values of each. We can again observe how each step in our pipeline successively improves the results.

\subsubsection{Evaluation of the User Interface}

To validate our hypothesis that the clusters generated by our method are similar, we created a user study that gave users the following task:

\section{Given an example asset, find 10 similar assets}

We measured the time taken by the users to search for the assets. Three methods were compared: 1 . The assets were randomly arranged. This is the baseline method over which we show considerable improvement. We call this method random. 2. In the second method, the assets are now clustered as explained in Section 3.5.5. The user is allowed to explore different clusters. This helps, because there are certain clusters where the user does not even perform a search, as the cluster style is different from the example. Note that since the clustering is not perfect, the user might select assets from multiple clusters. A perfect clustering would mean that only visually similar façades populate each cluster. This method is called cluster. 3. In the final method, instead of letting the user manually search within clusters, the user uploads the example asset to the UI and the UI returns the closest neighbours of the asset in the embedding space, as described in Section 3.5.5. We call this method search. For details of the complete experimental setup and the UI shown to the participants of the study, please refer to the Supplementary Material. 
TABLE 2: Ablation study to compare multiple variations of our framework. Column ' $K_{\text {vdirs' }}$ ' is the number candidate view directions used to estimate the $\mathrm{ZP}$, 'Consensus $\mathrm{ZP}$ ' is either the robust mean or robust SVD, ' $K_{\text {inlier }}$ ' is number of inlier points used in the consensus $Z P$ step, 'VP/ZP R' is checked if the viewing direction is refined after the initial $Z P / V P$ estimation, and 'Fac $R^{\prime}$ is checked if the façade rectification is re-estimated after initial façade detection.

\begin{tabular}{|c|c|c|c|c|c|c|c|c|}
\hline$K_{\text {vdirs }}$ & Consensus ZP & $K_{\text {inlier }}$ & $\mathrm{VP} / \mathrm{ZP} \mathrm{R}$ & Fac R & maa $\downarrow$ & mma $\downarrow$ & poa $\leq 1^{\circ} \uparrow$ & pom $\leq 2^{\circ} \uparrow$ \\
\hline 4 & Mean & 4 & & & $0.90^{\circ}$ & $1.90^{\circ}$ & $76.92 \%$ & $76.92 \%$ \\
\hline 8 & Mean & 4 & & & $0.66^{\circ}$ & $1.36^{\circ}$ & $84.62 \%$ & $78.85 \%$ \\
\hline 12 & Mean & 4 & & & $0.60^{\circ}$ & $1.24^{\circ}$ & $84.62 \%$ & $82.69 \%$ \\
\hline 16 & Mean & 4 & & & $0.57^{\circ}$ & $1.19^{\circ}$ & $88.46 \%$ & $82.69 \%$ \\
\hline 20 & Mean & 4 & & & $0.58^{\circ}$ & $1.20^{\circ}$ & $88.46 \%$ & $86.54 \%$ \\
\hline 4 & SVD & 4 & & & $0.76^{\circ}$ & $1.57^{\circ}$ & $78.85 \%$ & $76.92 \%$ \\
\hline 8 & SVD & 4 & & & $0.60^{\circ}$ & $1.29^{\circ}$ & $86.54 \%$ & $80.77 \%$ \\
\hline 12 & SVD & 4 & & & $0.60^{\circ}$ & $1.25^{\circ}$ & $84.62 \%$ & $82.69 \%$ \\
\hline 16 & SVD & 4 & & & $0.57^{\circ}$ & $1.19^{\circ}$ & $88.46 \%$ & $82.69 \%$ \\
\hline 20 & SVD & 4 & & & $0.57^{\circ}$ & $1.18^{\circ}$ & $88.46 \%$ & $86.54 \%$ \\
\hline 4 & Mean & 4 & $\checkmark$ & & $0.85^{\circ}$ & $1.77^{\circ}$ & $80.77 \%$ & $82.69 \%$ \\
\hline 8 & Mean & 4 & $\checkmark$ & & $0.64^{\circ}$ & $1.26^{\circ}$ & $88.46 \%$ & $84.62 \%$ \\
\hline 12 & Mean & 4 & $\checkmark$ & & $0.57^{\circ}$ & $1.18^{\circ}$ & $86.54 \%$ & $86.54 \%$ \\
\hline 16 & Mean & 4 & $\checkmark$ & & $0.56^{\circ}$ & $1.16^{\circ}$ & $90.38 \%$ & $88.46 \%$ \\
\hline 20 & Mean & 4 & $\checkmark$ & & $0.57^{\circ}$ & $1.21^{\circ}$ & $90.38 \%$ & $88.46 \%$ \\
\hline 4 & SVD & 4 & $\checkmark$ & & $0.72^{\circ}$ & $1.45^{\circ}$ & $82.69 \%$ & $80.77 \%$ \\
\hline 8 & SVD & 4 & $\checkmark$ & & $0.58^{\circ}$ & $1.21^{\circ}$ & $88.46 \%$ & $84.62 \%$ \\
\hline 12 & SVD & 4 & $\checkmark$ & & $0.57^{\circ}$ & $1.22^{\circ}$ & $88.46 \%$ & $84.62 \%$ \\
\hline 16 & SVD & 4 & $\checkmark$ & & $0.56^{\circ}$ & $1.14^{\circ}$ & $92.31 \%$ & $88.46 \%$ \\
\hline 20 & SVD & 4 & $\checkmark$ & & $0.56^{\circ}$ & $1.18^{\circ}$ & $90.38 \%$ & $86.54 \%$ \\
\hline 16 & SVD & 2 & $\bar{\checkmark}$ & & $0.56^{\circ}$ & $1.22^{\circ}$ & $88.46 \%$ & $84.62 \%$ \\
\hline 16 & SVD & 6 & $\checkmark$ & & $0.55^{\circ}$ & $1.14^{\circ}$ & $94.23 \%$ & $88.46 \%$ \\
\hline 16 & SVD & 8 & $\checkmark$ & & $0.56^{\circ}$ & $1.15^{\circ}$ & $88.46 \%$ & $88.46 \%$ \\
\hline 16 & SVD & 10 & $\checkmark$ & & $0.55^{\circ}$ & $1.12^{\circ}$ & $92.31 \%$ & $86.54 \%$ \\
\hline 16 & SVD & 12 & $\checkmark$ & & $0.53^{\circ}$ & $1.11^{\circ}$ & $92.31 \%$ & $88.46 \%$ \\
\hline 16 & SVD & 14 & $\checkmark$ & & $0.54^{\circ}$ & $1.12^{\circ}$ & $90.38 \%$ & $90.38 \%$ \\
\hline 16 & SVD & 16 & $\checkmark$ & & $0.60^{\circ}$ & $1.28^{\circ}$ & $82.69 \%$ & $84.62 \%$ \\
\hline 16 & SVD & 12 & $\checkmark$ & $\checkmark$ & $0.47^{\circ}$ & $0.98^{\circ}$ & $\mathbf{9 4 . 2 3} \%$ & $\mathbf{9 0 . 3 8} \%$ \\
\hline
\end{tabular}

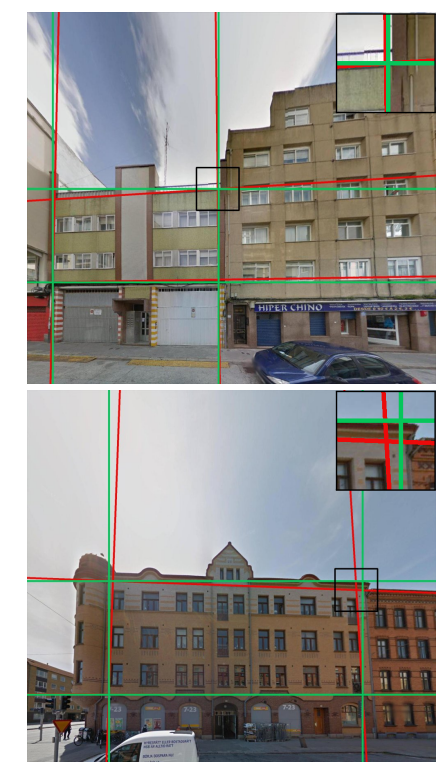

(a) GIS

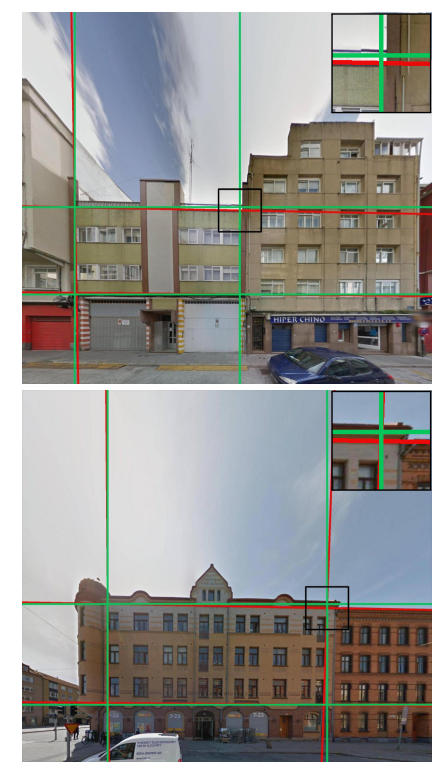

(b) VP/ZP E

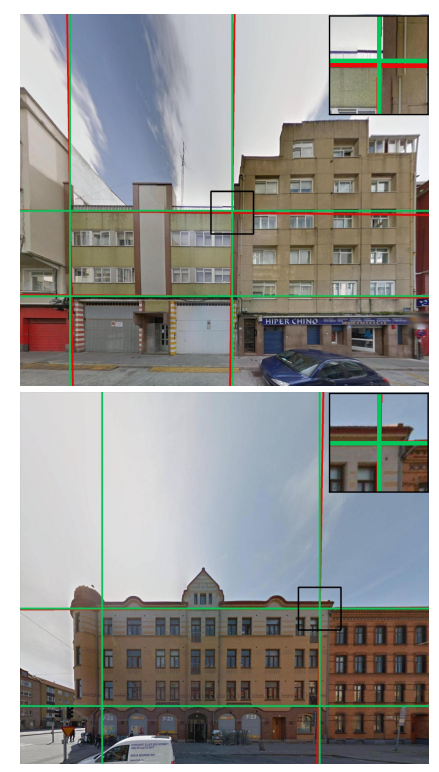

(c) $\mathrm{VP} / \mathrm{ZP} E+\mathrm{R}$

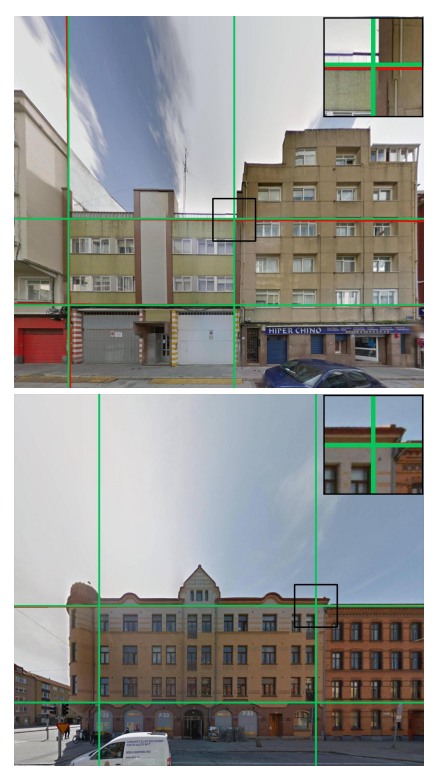

(d) VP/ZP E+R + Fac R

Fig. 10: Rectification Examples. (a) Projection from the plane described by GIS data (b) Projection using eight view directions and four inlier points for the consensus ZP. (c) Projection using 16 view directions and 12 inlier points for the consensus $\mathrm{ZP}$, also including refinement of the ZP. (d) Adds façade rectification to (c). 
TABLE 3: Detection results for façades. We compare three algorithms: Mask RCNN (M-RCNN), Faster RCNN with default anchor aspect ratios (F-RCNN), and Faster RCNN with customized anchor aspect ratios $\left(\mathrm{F}-\mathrm{RCNN}^{*}\right)$. The label setting $\mathrm{F}$ means we only detect façades, while $\mathrm{F}+\mathrm{W}$ means we detect façades and windows jointly. We compute the metrics AP with parameter IoU and AR with parameter maximum detections $\left(\Delta_{n}\right)$.

\begin{tabular}{lcccc|cc}
\hline \multirow{2}{*}{ Method } & \multirow{2}{*}{ Label } & \multicolumn{3}{c|}{ AP (IoU) } & \multicolumn{2}{c}{ AR $\left(\Delta_{n}\right)$} \\
\cline { 3 - 7 } & & $50: 95$ & 50 & 75 & 1 & 10 \\
\hline F-RCNN & \multirow{2}{*}{48.1} & 67.3 & 53.1 & 38.5 & 59.8 \\
F-RCNN* & F & 48.4 & 67.6 & 52.9 & $\mathbf{3 8 . 6}$ & $\mathbf{6 0 . 2}$ \\
M-RCNN & & 45.6 & 63.6 & 50.7 & 35.2 & 57.5 \\
\hline F-RCNN* & \multirow{2}{*}{ F+W } & $\mathbf{5 1 . 1}$ & $\mathbf{7 1 . 6}$ & $\mathbf{5 6 . 7}$ & 38.0 & 60.1 \\
M-RCNN & & 45.0 & 69.0 & 47.1 & 37.3 & 56.9 \\
\hline
\end{tabular}

TABLE 4: Detection results for windows. The setting $\mathrm{W}$ on I refers to detecting windows on rectified images and $W$ on $F$ means detecting windows on rectified images cropped to façades using façade detection results. See Table 3 for an explanation of the metrics.

\begin{tabular}{lcccc|cc}
\hline \multirow{2}{*}{ Method } & \multirow{2}{*}{ Label } & \multicolumn{3}{c|}{$\mathrm{AP}(\mathrm{IoU})$} & \multicolumn{2}{c}{$\mathrm{AR}\left(\Delta_{n}\right)$} \\
\cline { 3 - 7 } & & $50: 95$ & 50 & 75 & 10 & 100 \\
\hline F-RCNN* & \multirow{2}{*}{ W on I } & 28.7 & 54.0 & 27.6 & 14.5 & 36.5 \\
M-RCNN & & 28.3 & 53.0 & 27.2 & 14.5 & 36.0 \\
\hline F-RCNN* & \multirow{2}{*}{ W on F } & 33.4 & $\mathbf{6 4 . 8}$ & 31.9 & 23.2 & $\mathbf{4 4 . 3}$ \\
M-RCNN & & $\mathbf{3 3 . 4}$ & 62.9 & $\mathbf{3 3 . 0}$ & $\mathbf{2 3 . 5}$ & 43.8 \\
\hline
\end{tabular}

TABLE 5: Comparing the impact of rectification algorithms on façade detection results using Faster RCNN. Random - randomly choosing four view directions and picking the best one. Fixed - taking four evenly spaced view directions. GIS - taking view directions pointing towards façades in GIS data. VP/ZP E - our initial horizon and VP estimation; $\mathrm{VP} / \mathrm{ZP} \mathrm{E}+\mathrm{R}$ - our complete framework.

\begin{tabular}{lcc|cc}
\hline \multirow{2}{*}{ Method } & \multicolumn{2}{c|}{ AP $(\mathrm{IoU})$} & \multicolumn{2}{c}{ AR $\left(\Delta_{n}\right)$} \\
\cline { 2 - 5 } & $50: 95$ & 75 & 1 & 10 \\
\hline Random & 9.3 & 9.2 & 9.0 & 11.9 \\
Fixed & 42.8 & 50.7 & 36.1 & 56.0 \\
GIS & 47.7 & 57.2 & 36.3 & 58.1 \\
VP/ZP E & 49.0 & 56.7 & 37.0 & 58.8 \\
VP/ZP E + R & $\mathbf{5 0 . 4}$ & $\mathbf{5 8 . 6}$ & $\mathbf{3 8 . 8}$ & $\mathbf{6 0 . 8}$ \\
\hline
\end{tabular}

TABLE 6: Comparing the impact of rectification algorithms on window detection results using Mask RCNN. See Table 5 for a detailed description.

\begin{tabular}{lcc|cc}
\hline \multirow{2}{*}{ Method } & \multicolumn{2}{c|}{ AP $(\mathrm{IoU})$} & \multicolumn{2}{c}{ AR $\left(\Delta_{n}\right)$} \\
\cline { 2 - 5 } & $50: 95$ & 50 & 10 & 100 \\
\hline Random & 11.5 & 20.5 & 5.8 & 12.9 \\
Fixed & 27.5 & 52.7 & 14.1 & 35.2 \\
GIS & 28.1 & 53.2 & 14.1 & 35.5 \\
VP/ZP E & $\mathbf{2 8 . 7}$ & 54.8 & 14.4 & 36.2 \\
VP/ZP E + R & 28.5 & $\mathbf{5 5 . 4}$ & $\mathbf{1 4 . 7}$ & $\mathbf{3 6 . 7}$ \\
\hline
\end{tabular}
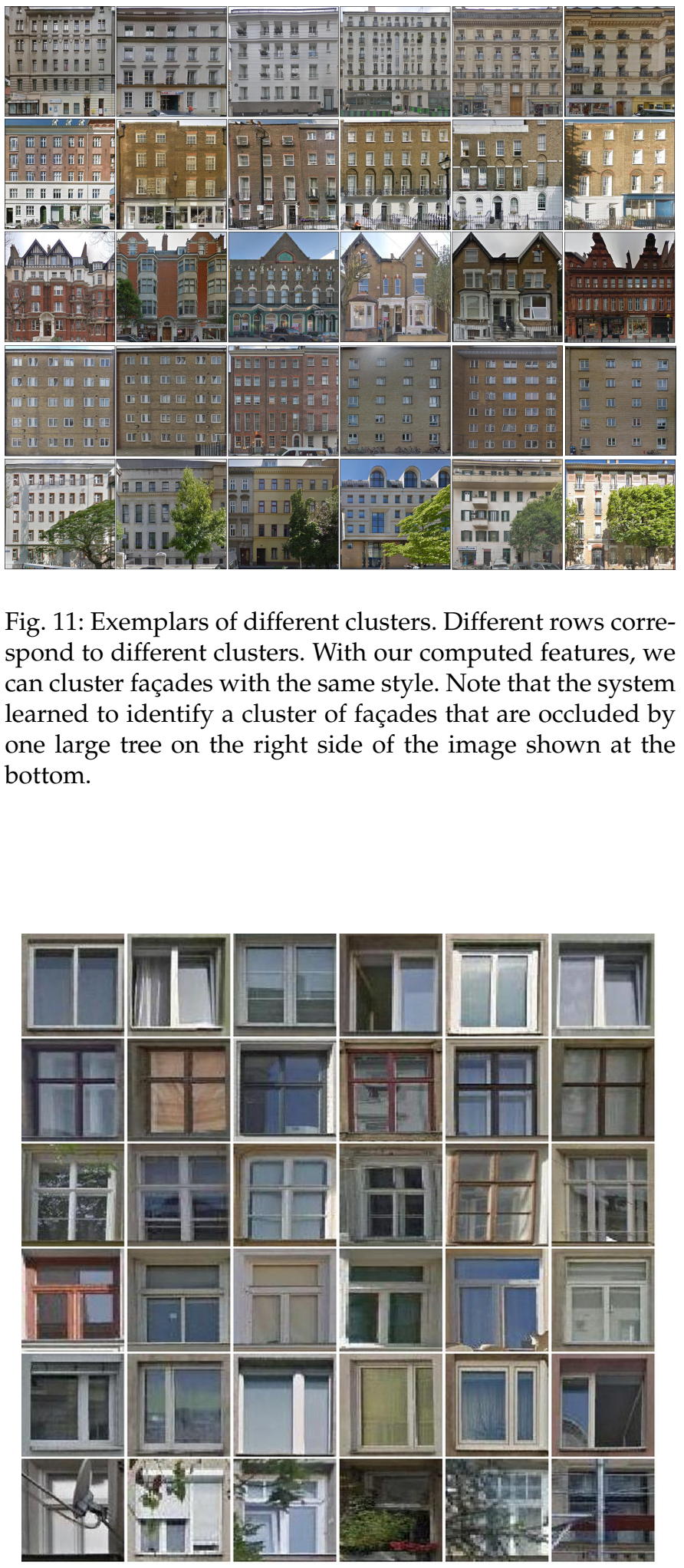

Fig. 11: Exemplars of different clusters. Different rows correspond to different clusters. With our computed features, we can cluster façades with the same style. Note that the system learned to identify a cluster of façades that are occluded by one large tree on the right side of the image shown at the bottom.

Fig. 12: Exemplars of different clusters. Different rows correspond to different window clusters. The first five rows depict clusters of windows with the same window frame structure. The last row depicts a cluster of occluded windows. 
To perform the user-study, we selected window as the asset over which the search was to be performed, as different window styles were readily apparent. We randomly chose a subset containing 546 windows. 15 non-expert users participated in the user-study. Non-expert means that the users did not have any experience in architecture or urban modeling. Each user was allowed to choose three images from the 546 images and search for ten assets that were similar to the chosen image. The user performed all three types of search in the following order: random, cluster, search, and the time taken for the search was recorded. The details of the study are shown in Fig. 13.

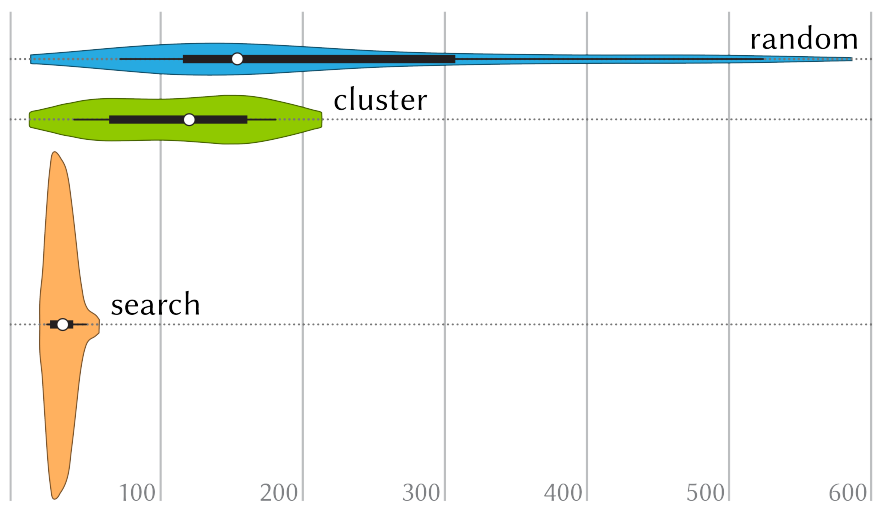

Fig. 13: Violin plots of the amount of time taken to search for an asset. The $y$-axis is the method used to perform the search. The $x$-axis is the time in seconds. We see that random has a median at about 150 s, but with a very long tail corresponding to window styles that are rare and cannot be easily found using a random search. cluster improves the median time just above 100s and reduces the long-tail of the distribution in random. When using search, users took about 30s to perform searches. This was achieved regardless of the rarity of the exemplar window, as is clear from the very sharp peak in the distribution.

\subsection{Qualitative Evaluation}

Here, we describe qualitative results and demonstrate applications that enabled the extracted assets. We used our framework to download 78,377 panoramas of size 13312 by 6656 pixels. We extracted and rectified 213,485 views to be used by the object detection networks. In total, we extracted 199,723 façades. We filtered these façades automatically, using the filtering capability in our UI, to select a higher quality subset of about $23 \mathrm{~K}$ façades. We only extracted those that contained architectural elements for this subset: 516K windows, $28 \mathrm{~K}$ doors, and $142 \mathrm{~K}$ balconies. We considered a façade to be high quality if it had low blur, low occlusion, and large resolution.

\subsubsection{Clustering And Visualization to Explore Architectural Styles}

We found that clustering is an effective tool to structure and analyze the extracted data, as supported by a user study presented in Fig. 13. In Fig. 11, we visualize extracted façades according to the clustering of the data and in Figs. 12

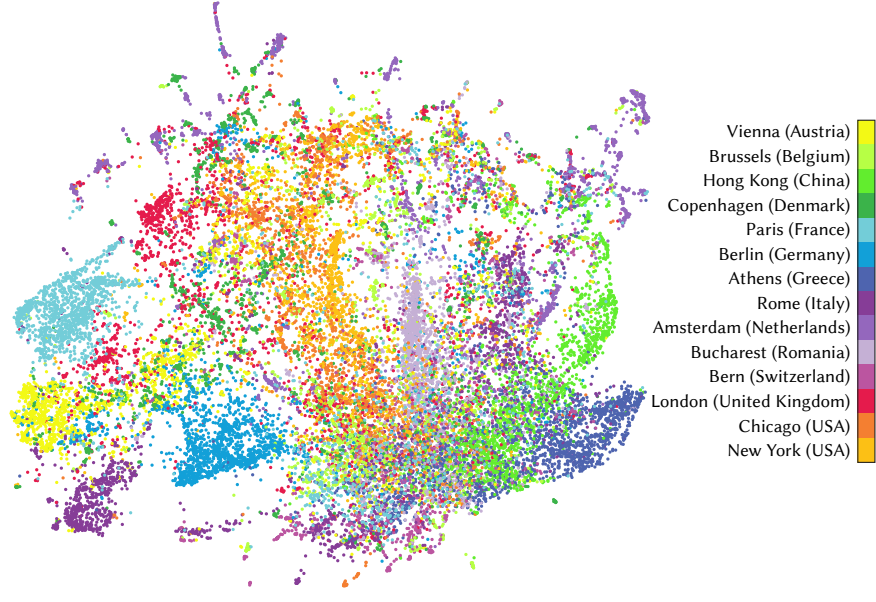

Fig. 14: A visualization of all the façades in our dataset. This 2D embedding computed using our pipeline seems to respect geographic distance to some extent.

and 18, we visualize window clusters. From Figs. 12 and 18, we observe that there is a lot of variability in the façade data, but we can see that the clusters contain façades of similar appearance and structure. The window clusters have less variability, and clusters often correspond to windows with identical frame structure. In Fig. 14, we show a 2D embedding of extracted façades. We can observe that many façades are close to other façades in the same city, indicating that the embedding captures some architectural coherence within those cities. Also, we can observe that most cities have façades appearing in multiple clusters, hinting at different dominant styles within those cities. We can also observe that styles are more similar between cities that are geographically close to each other, e.g., we can see that many of the façades from Vienna are similar to both German and Italian façades (Fig. 14 bottom-left corner), which reflects the fact that Austria borders both Germany and Italy. In Fig. 19, we show a cluster visualization showing the distribution of façade styles on a map of London. We observe that the styles are well mixed overall, but there is a coherence of style in certain neighborhoods.

\subsubsection{Locating Assets}

Since our assets include latitude and longitude information, we can use extracted assets for geospatial localization. For example, a designer might want to model a detailed asset as 3D geometry, e.g., a façade or a window, and obtain accurate $3 \mathrm{D}$ scans as a template. They could query a given style that they are interested in and our search would then give possible locations where similar elements are present nearby. We visualize such a spatial façade search in Fig. 15.

\subsubsection{Synthesizing Façade Layouts Using GANs}

To evaluate the usefulness of the extracted elements for data-driven modeling, we train multiple GANs. In Fig. 16, we show selected façades and windows from training progressive GANs [23] with $30 \mathrm{~K}$ and $150 \mathrm{~K}$ images, respectively. The GAN is able to produce a large number of façades of good visual quality. We also trained SPADE [60], which can take a label map as input and produce an RGB image as 


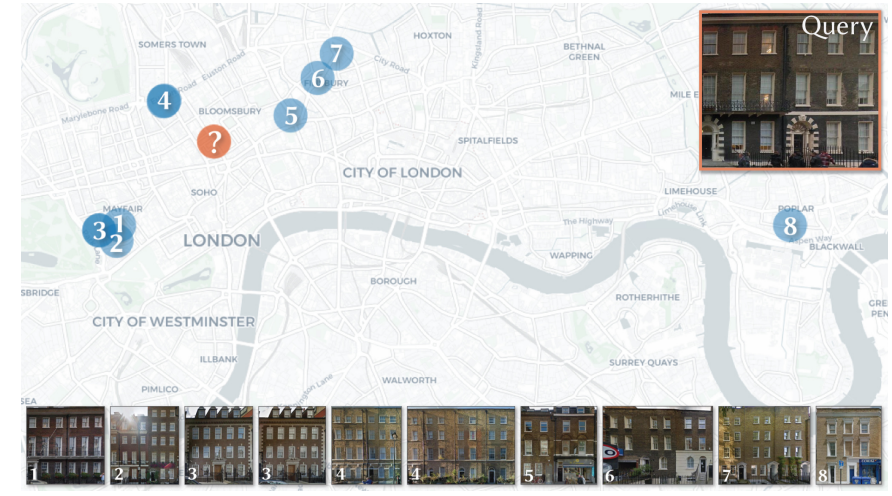

Fig. 15: We show the location of the query façade (represented in orange) and the locations of the nearest neighbors (in blue). The search is performed in the 200-dimensional embedding space. As we store geographic metadata about our assets, neighbors that are close in the embedding space, but not close geographically, can be filtered out.

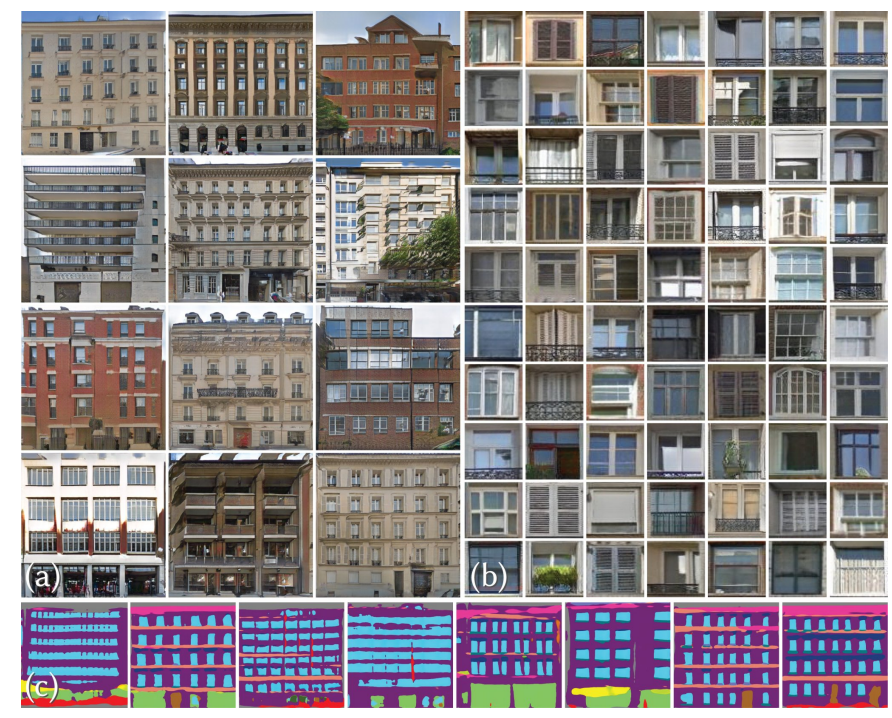

Fig. 16: We show results synthesized using ProGAN on the assets we extracted. In (a), we show generated façades that demonstrate that we capture a large variety of façade styles, which can be replicated by the trained network. (b) depicts a collection of generated windows, showcasing the multitude of styles and lighting situations that are contained within our assets. In (c), we show results generated by a GAN trained on segmented façade layouts.

output. We use the semantic segmentation results of Section 3.5.3 to generate semantic labels for each façade image when training SPADE. In Fig. 17, we extract segmentation masks from existing façade images and compare SPADE results to the corresponding real images.

Finally, we can synthesize façades indirectly, by training a progressive GAN on segmentation masks and then using SPADE on the results to produce RGB images (See Fig. 20). We provide an interactive UI to produce façade images from segmentation masks, as shown in Fig. 21 and the accompanying video.

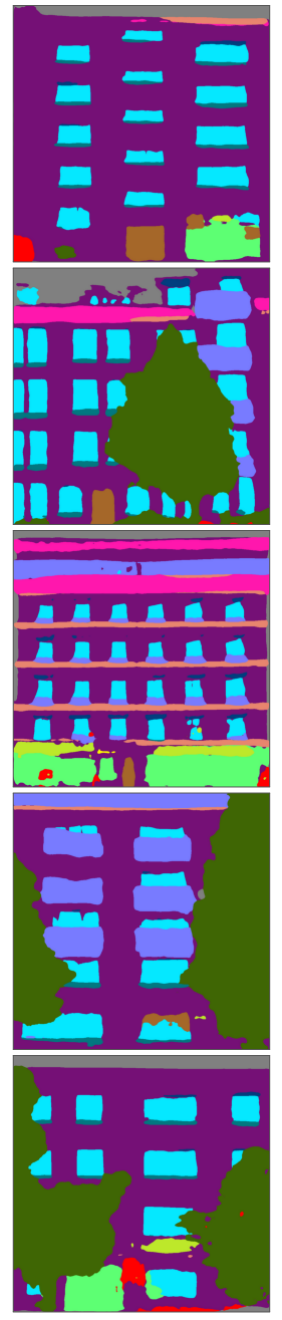

(a) Label Mask
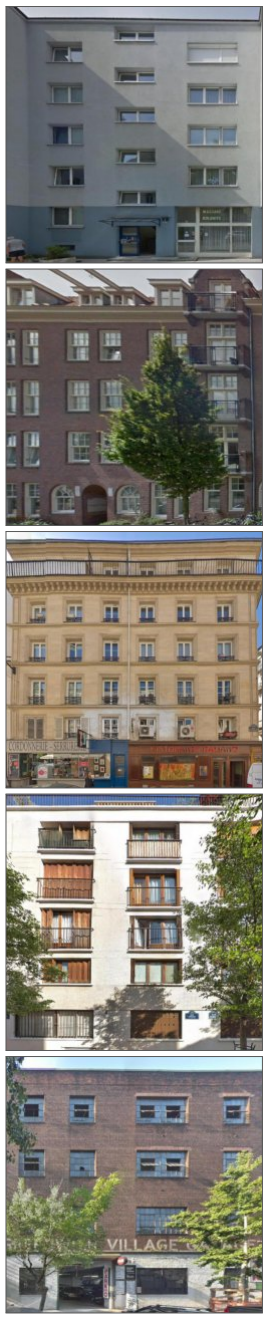

(b) Real

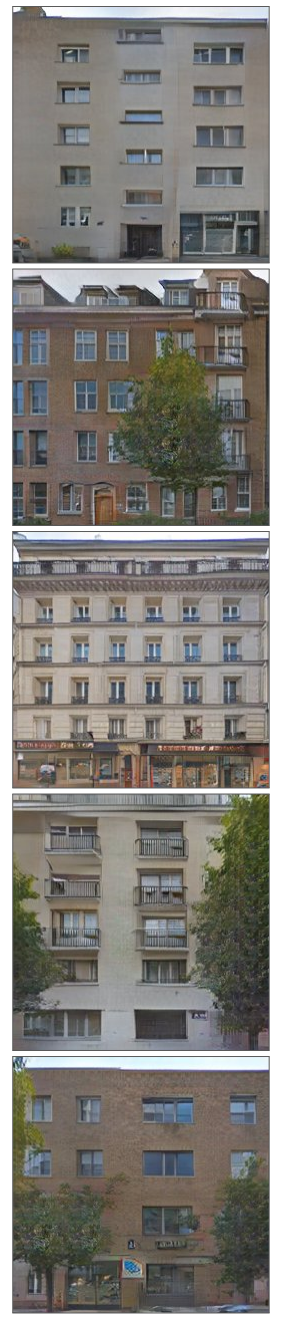

(c) Synthesized
Fig. 17: Images synthesized by SPADE: input segmentation mask (left), original image used to extract the segmentation mask (middle), and synthesized façade image (right).

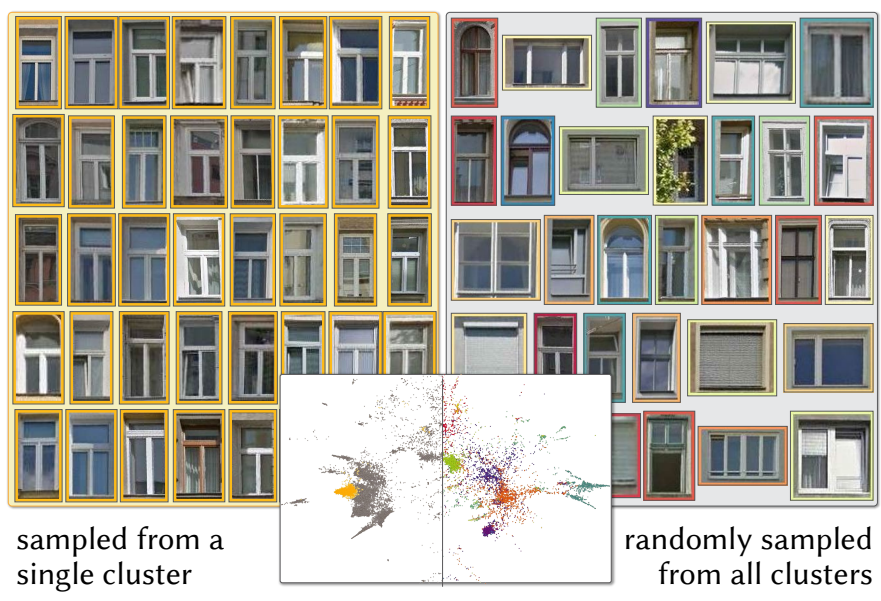

Fig. 18: We show how a sampling of assets (here windows) differs when random sampling from a single cluster (left) compared to a diverse random sampling from all clusters (right). 


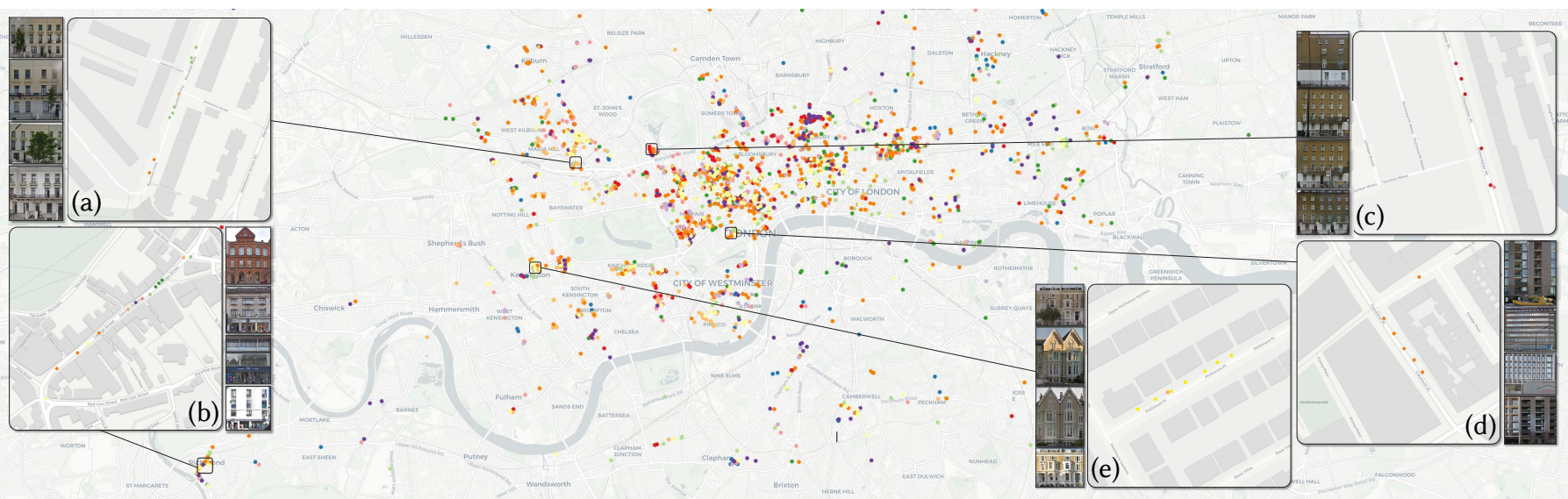

Fig. 19: We show the distribution of different façade types in London. While the façade types are well mixed on the overview map, we can often see clusters of similar façades within local neighborhoods or streets $(\mathrm{a}, \mathrm{c}, \mathrm{d}, \mathrm{e})$. Some streets also exhibit a wide range of façade styles (b). Insets show selected façade images.

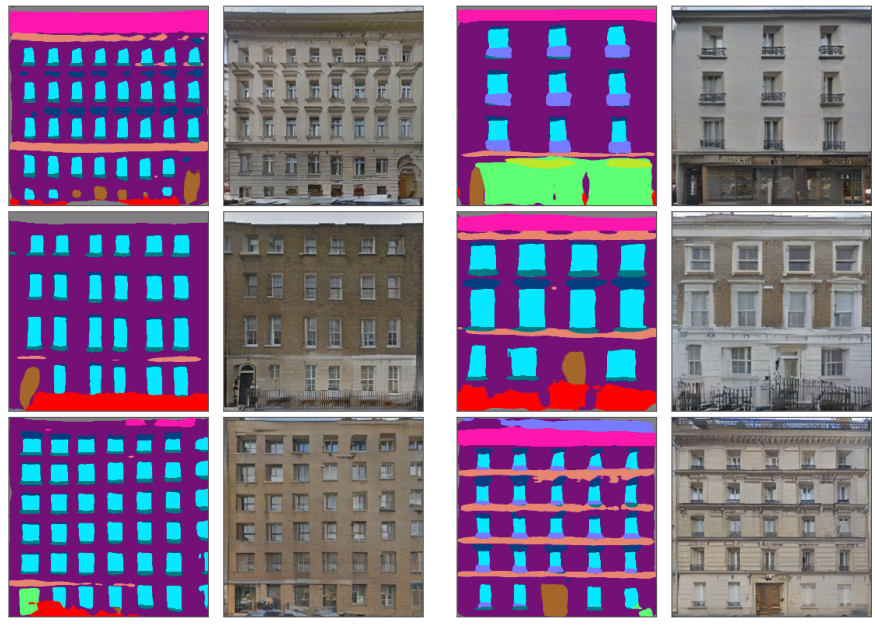

Fig. 20: Selected façade layouts synthesized by a GAN trained on segmentation masks and translations to images computed by SPADE. Notice that the segmentation mask, encoding the layout, and the generated façade are not real.

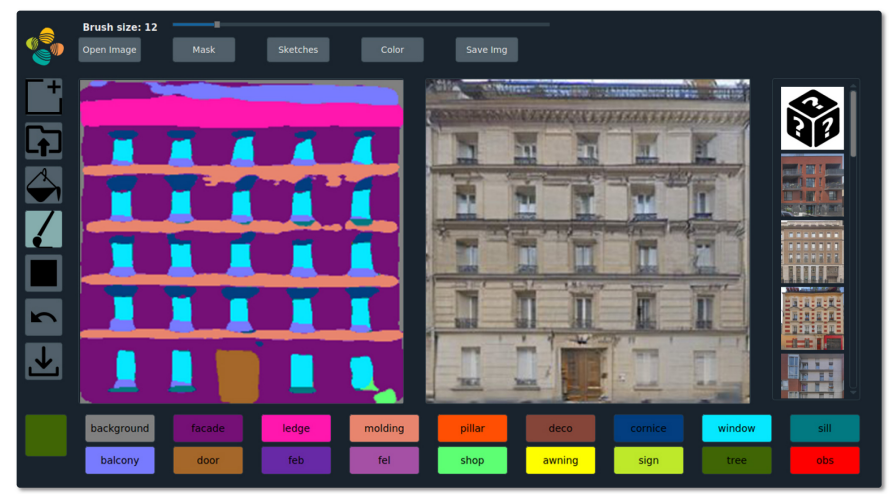

Fig. 21: A UI facilitates an interactive synthesis of façades from segmentation layouts. Users can paint architectural elements on the segmentation mask on the left and the generated façades updates interactively.

\subsection{Discussion}

We noticed multiple limitations in our framework. First, the quality of the collected data varies and data is not available for all parts of the world. That means that although our framework enables the architectural analysis of cities and regions that are well covered, it can only provide a biased analysis of architecture worldwide. Second, currently available panoramic images have various occlusion, stitching, and dynamic range artifacts. As a result, the image quality of automatically acquired data does not match the quality of carefully acquired images. In future work, it might be useful to capture more dense video data of cameras pointed directly towards façades while omitting parts of the sky and the road. Third, our object detection framework only uses about a thousand annotated panoramic images. While our object detection results are good in general, we also noticed multiple shortcomings with our test data. Thus, we would like to explore the impact of improved annotations in future work. The system described here might be a useful tool in selecting which assets to annotate.

Another main issue is that the concept of façade is somewhat ill-defined and that annotators find it difficult to mark façades consistently. For example, it is unclear if flags or antennas attached to façades should be included in the annotation and it is often unclear where the façade ends and the roof begins. In addition, perspective distortions of overhanging façade elements near the roof leads to bounding box boundaries that do not tightly contain wall boundaries. The assumption that a façade has a single dominant plane is often correct; however, many buildings include bays or protruding and recessed architectural elements. Many buildings are comprised of multiple parts, with upper levels set back from the lower levels, making it hard to group planes into a single façade. Conversely, many adjacent buildings share a plane and even some design similarities, so it is difficult to train annotators to consistently distinguish between them. A similar problem exists for windows; it can be difficult to determine if the boundary needs to consistently include the frame and decorations such as pediments or window sills. It can also be difficult to 
determine if a group of windows counts as multiple distinct objects, or a single object with three panels.

In future work, we would like to extract regularized structural information and material information from the façades and windows. Furthermore, we would like to use this information as prior information for building reconstruction from a single image. Finally, we are interested in building GANs that operate on façade and window structures rather than images.

\section{CONCLUSION}

We presented a system to extract architectural assets from large-scale collections of panoramic imagery. We proposed a new framework for automatically rectifying, detecting, and cropping image regions that contain architectural elements such as façades, windows, doors, or balconies. We computed various attributes for the assets and proposed a UI to visualize and query assets. Our framework has many applications in urban modeling, urban reconstruction, data collection for machine learning, architectural analysis, and geospatial localization, which we demonstrate for some selected applications. In total, we were able to extract a database of 199,723 façade images and images of architectural elements that these façades contain.

\section{ACKNOWLEDGEMENTS}

We thank the anonymous reviewers for their helpful comments. This work was supported by the KAUST Office of Sponsored Research (OSR) under Award No. OSRCRG2018-3730, OSR-2019-CPF-4102.3, and the Visual Computing Center at KAUST.

\section{REFERENCES}

[1] J. Zhu, R. Zhang, D. Pathak, T. Darrell, A. A. Efros, O. Wang, and E. Shechtman, "Toward multimodal image-to-image translation," CoRR, 2017

[2] X. Huang, M. Liu, S. J. Belongie, and J. Kautz, "Multimodal unsupervised image-to-image translation," CoRR, 2018.

[3] A. Brock, J. Donahue, and K. Simonyan, "Large scale GAN training for high fidelity natural image synthesis," ICLR, 2019.

[4] T. Karras, S. Laine, and T. Aila, "A style-based generator architecture for generative adversarial networks," IEEE CVPR, 2019.

[5] B. Fröhlich, E. Rodner, and J. Denzler, "A fast approach for pixelwise labeling of facade images," in ICPR, 2010.

[6] R. Tyleček and R. Śára, "Spatial pattern templates for recognition of objects with regular structure," in GCPR, 2013.

[7] G. Simon, A. Fond, and M.-O. Berger, "A-Contrario Horizon-First vanishing point detection using Second-Order grouping laws," in ECCV, 2018.

[8] S. Ren, K. He, R. Girshick, and J. Sun, "Faster R-CNN: Towards Real-Time object detection with region proposal networks," IEEE transactions on pattern analysis and machine intelligence, 2017.

[9] C. Doersch, S. Singh, A. Gupta, J. Sivic, and A. A. Efros, "What makes paris look like paris?" ACM Trans. Graph. (SIGGRAPH), 2012.

[10] J.-Y. Zhu, Y. J. Lee, and A. A. Efros, "Averageexplorer: Interactive exploration and alignment of visual data collections," ACM Trans. Graph. (SIGGRAPH), 2014.

[11] X. Chen, A. Shrivastava, and A. Gupta, "Neil: Extracting visual knowledge from web data," in IEEE ICCV 2013.

[12] D. J. Crandall, L. Backstrom, D. Huttenlocher, and J. Kleinberg, "Mapping the world's photos," in ACM WWW, 2009.

[13] B. Zhou, L. Liu, A. Oliva, and A. Torralba, "Recognizing city identity via attribute analysis of geo-tagged images," in ECCV, 2014.
[14] S. M. Arietta, A. A. Efros, R. Ramamoorthi, and M. Agrawala, "City forensics: Using visual elements to predict non-visual city attributes," IEEE Transactions on Visualization and Computer Graphics, vol. 20, no. 12, Dec 2014.

[15] A. Dubey, N. Naik, D. Parikh, R. Raskar, and C. A. Hidalgo, "Deep learning the city: Quantifying urban perception at a global scale," in $E C C V, 2016$.

[16] C. A. Vanegas, D. G. Aliaga, and B. Benes, "Building reconstruction using manhattan-world grammars," in IEEE CVPR, 2010.

[17] B. Watson, P. Müller, O. Veryovka, A. Fuller, P. Wonka, and C. Sexton, "Procedural urban modeling in practice," IEEE computer graphics and applications, vol. 28, no. 3, May 2008.

[18] Y. I. H. Parish and P. Müller, "Procedural modeling of cities," in ACM Trans. Graph. (SIGGRAPH), 2001.

[19] O. Št'ava, B. Beneš, R. Měch, D. G. Aliaga, and P. Krištof, "Inverse procedural modeling by automatic generation of l-systems," Computer Graphics Forum (Eurographics), 2010.

[20] İ. Demir, D. G. Aliaga, and B. Benes, "Proceduralization for editing 3D architectural models," in 3DV, 2016.

[21] C.-H. Shen, S.-S. Huang, H. Fu, and S.-M. Hu, "Adaptive partitioning of urban facades," ACM Trans. Graph., vol. 30, no. 6, p. 184, 2011.

[22] G. Nishida, A. Bousseau, and D. G. Aliaga, "Procedural modeling of a building from a single image," Computer Graphics Forum (Eurographics), 2018.

[23] T. Karras, T. Aila, S. Laine, and J. Lehtinen, "Progressive growing of gans for improved quality, stability, and variation," ICLR, 2018.

[24] P. Isola, J. Zhu, T. Zhou, and A. A. Efros, "Image-to-image translation with conditional adversarial networks," CoRR, 2016.

[25] P. Zhu, R. Abdal, Y. Qin, and P. Wonka, "Sean: Image synthesis with semantic region-adaptive normalization," 2019.

[26] H. Su, C. R. Qi, Y. Li, and L. J. Guibas, "Render for cnn: Viewpoint estimation in images using cnns trained with rendered $3 \mathrm{~d}$ model views," in IEEE ICCV, 2015.

[27] B. Tekin, S. N. Sinha, and P. Fua, "Real-Time Seamless Single Shot 6D Object Pose Prediction," in IEEE CVPR, 2018.

[28] Y. Xiang, T. Schmidt, V. Narayanan, and D. Fox, "Posecnn: A convolutional neural network for $6 \mathrm{~d}$ object pose estimation in cluttered scenes," 2018.

[29] F. Han and S.-C. Zhu, "Bottom-up/Top-Down image parsing by attribute graph grammar," in IEEE ICCV 2005.

[30] — "Bottom-up/top-down image parsing with attribute grammar," IEEE transactions on pattern analysis and machine intelligence, vol. 31, no. 1, Jan. 2009.

[31] B. Micusik, H. Wildenauer, and J. Kosecka, "Detection and matching of rectilinear structures," in IEEE CVPR, 2008.

[32] A. Fond, M.-O. Berger, and G. Simon, "Facade Proposals for Urban Augmented Reality," in ISMAR 2017 - 16th IEEE International Symposium on Mixed and Augmented Reality, Nantes, France, Oct. 2017.

[33] R. Girshick, J. Donahue, T. Darrell, and J. Malik, "Rich feature hierarchies for accurate object detection and semantic segmentation," in IEEE CVPR, 2014.

[34] K. He, G. Gkioxari, P. Dollar, and R. Girshick, "Mask r-cnn," in IEEE ICCV, 2017.

[35] L. Affara, L. Nan, B. Ghanem, and P. Wonka, "Large scale asset extraction for urban images," in ECCV, 2016.

[36] C. Wu, J.-M. Frahm, and M. Pollefeys, "Detecting large repetitive structures with salient boundaries," in ECCV, 2010.

[37] J. Košecká and W. Zhang, "Extraction, matching, and pose recovery based on dominant rectangular structures," Computer vision and image understanding: CVIU, vol. 100, no. 3, Dec. 2005.

[38] J. Tardif, "Non-iterative approach for fast and accurate vanishing point detection," in IEEE ICCV, 2009.

[39] H. Wildenauer and A. Hanbury, "Robust camera self-calibration from monocular images of manhattan worlds," in IEEE CVPR, 2012.

[40] E. Tretyak, O. Barinova, P. Kohli, and V. Lempitsky, "Geometric image parsing in Man-Made environments," International journal of computer vision, vol. 97, no. 3, May 2012.

[41] Y. Xu, S. Oh, and A. Hoogs, "A minimum error vanishing point detection approach for uncalibrated monocular images of ManMade environments," in IEEE CVPR, 2013.

[42] M. Zhai, S. Workman, and N. Jacobs, "Detecting vanishing points using global image context in a non-manhattan world," in IEEE CVPR, 2016. 
[43] F. Kluger, H. Ackermann, M. Y. Yang, and B. Rosenhahn, “Deep learning for vanishing point detection using an inverse gnomonic projection," in Pattern Recognition, 2017.

[44] R. Grompone von Gioi, J. Jakubowicz, J.-M. Morel, and G. Randall, "LSD: a line segment detector," Image Processing On Line, vol. 2, Mar. 2012.

[45] J.-C. Bazin, C. Demonceaux, P. Vasseur, and I. Kweon, "Rotation estimation and vanishing point extraction by omnidirectional vision in urban environment," The International Journal of Robotics Research, vol. 31, no. 1, pp. 63-81, 2012.

[46] D. Anguelov, C. Dulong, D. Filip, C. Frueh, S. Lafon, R. Lyon, A. Ogale, L. Vincent, and J. Weaver, "Google street view: Capturing the world at street level," Computer, vol. 43, no. 6, 2010.

[47] A. Desolneux, L. Moisan, and J.-M. Morel, From gestalt theory to image analysis: a probabilistic approach. Springer Science \& Business Media, 2007, vol. 34

[48] J. Femiani, W. R. Para, N. J. Mitra, and P. Wonka, "Facade segmentation in the wild," CoRR, 2018.

[49] T. Kelly, J. Femiani, P. Wonka, and N. J. Mitra, "Bigsur: Large-scale structured urban reconstruction," ACM Trans. Graph., vol. 36, no. 6, November 2017.

[50] K. He, X. Zhang, S. Ren, and J. Sun, “Deep residual learning for image recognition," in IEEE CVPR, 2016.

[51] T.-Y. Lin, P. Dollar, R. Girshick, K. He, B. Hariharan, and S. Belongie, "Feature pyramid networks for object detection," in IEEE CVPR, 2017.

[52] M. Caron, P. Bojanowski, A. Joulin, and M. Douze, "Deep clustering for unsupervised learning of visual features," in ECCV, 2018.

[53] L.-C. Chen, Y. Zhu, G. Papandreou, F. Schroff, and H. Adam, "Encoder-decoder with atrous separable convolution for semantic image segmentation," in ECCV, 2018.

[54] T. Kelly, P. Guerrero, A. Steed, P. Wonka, and N. J. Mitra, “Frankengan: Guided detail synthesis for building mass models using stylesynchonized gans," ACM Trans. Graph., vol. 37, no. 6, Dec. 2018.

[55] S. Pertuz, D. Puig, and M. A. Garcia, "Analysis of focus measure operators for shape-from-focus," Pattern recognition, vol. 46, no. 5, May 2013.

[56] K. Simonyan and A. Zisserman, "Very Deep Convolutional Networks for Large-Scale Image Recognition," ArXiv e-prints, 2014.

[57] L. McInnes and J. Healy, "UMAP: Uniform Manifold Approximation and Projection for Dimension Reduction," ArXiv e-prints, 2018

[58] N. Snavely, S. M. Seitz, and R. Szeliski, "Photo tourism: Exploring photo collections in 3D," ACM Trans. Graph., vol. 25, no. 3, Jul. 2006.

[59] J. Shotton, B. Glocker, C. Zach, S. Izadi, A. Criminisi, and A. Fitzgibbon, "Scene coordinate regression forests for camera relocalization in RGB-D images," in IEEE CVPR, 2013.

[60] T. Park, M.-Y. Liu, T.-C. Wang, and J.-Y. Zhu, "Semantic image synthesis with spatially-adaptive normalization," in IEEE CVPR, 2019.

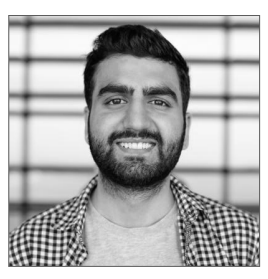

Wamiq Reyaz Para is a PhD Student of Computer Science at the Visual Computing Center at King Abdullah University of Science and Technology (KAUST).

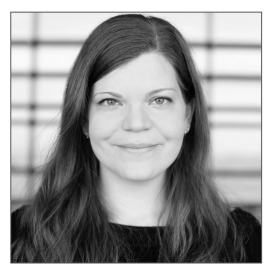

Anna Frühstück is a PhD Student of Computer Science at the Visual Computing Center at King Abdullah University of Science and Technology (KAUST). Her research interests include image synthesis and generative modeling techniques as well as machine learning techniques for Computer Graphics and Computer Vision.

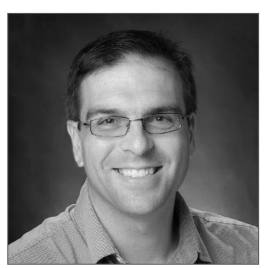

John Femiani is Associate Professor in Computer Science and Software Engineering at $\mathrm{Mi}-$ ami University in Ohio. He received his doctorate in Computer Science from Arizona State University. After his $\mathrm{PhD}$, he worked as assistant professor at ASU Polytechnic and was CTO for VProctor Inc. His research interests include remote sensing, computer vision, computer graphics, and deep learning.

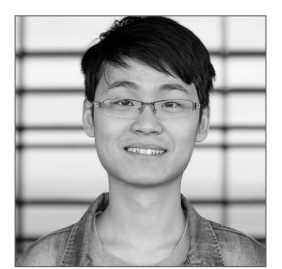

Peihao Zhu is a PhD Student of Computer Science at the Visual Computing Center at King Abdullah University of Science and Technology (KAUST). His research interests include Computer Vision and Computer Graphics.

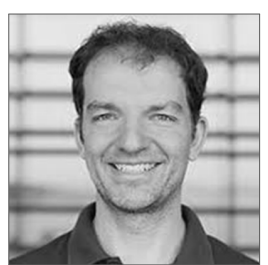

Peter Wonka is Full Professor in Computer Science at King Abdullah University of Science and Technology (KAUST) and Associate Director of the Visual Computing Center (VCC). His research interests include various topics in deep learning, computer graphics, computer vision, remote sensing, image processing, and visualization. 